\title{
Feedback-Aided Pilot Placement and Subcarrier Pairing for AF OFDM Relay Channels
}

\author{
Kuang-Yu Sung, Student Member, IEEE, Y.-W. Peter Hong, Member, IEEE, and Chi-Chao Chao, Member, IEEE
}

\begin{abstract}
This paper examines the use of channel feedback to adaptively determine pilot placement among subcarriers in amplify-and-forward (AF) orthogonal frequency-division multiplexing (OFDM) relay systems. With feedback of the previous channel estimate, pilot subcarriers at the source and relay can be reassigned to reduce channel estimation error and ensure that good subcarriers are made available for data transmission. The minimum effective signal-to-noise ratio (SNR) among data subcarriers is utilized as the performance metric for the optimization of the pilot locations. The effective SNR is defined as the receive SNR at the destination, taking into consideration the source-to-relay (SR) and relay-to-destination (RD) channels, as well as their respective channel estimation errors. The feedback gain, which is defined as the ratio between the minimum effective SNR achieved with the optimal feedback-aided pilot placement and that achieved with the equal-spaced pilot placement, is used to measure the gains achieved through feedback. In this paper, we first derive an analytic lower bound on the optimal feedback gain and show its rate of increase with respect to the number of subcarriers. These studies are performed for systems both without and with subcarrier pairing (SP) at the relay. Specifically, in systems without $\mathrm{SP}$, the relay forwards each datum on the same subcarrier on which it is received. In systems with SP, the relay is allowed to reassign the subcarriers used to forward the data received from the source. With the goal of maximizing the minimum effective SNR, the optimal SP scheme is shown to be an inverse mapping between the SR and RD subcarriers with respect to their channel gains. Due to the complexity of finding the optimal placement, a suboptimal but efficient algorithm called the iterative pilot relocation (IterPR) scheme is then proposed. The effectiveness of the proposed IterPR schemes is demonstrated through numerical simulations.
\end{abstract}

Index Terms-Channel estimation, feedback, orthogonal frequency-division multiplexing (OFDM), pilot placement, relaying, subcarrier pairing $(\mathrm{SP})$.

\section{INTRODUCTION}

$\mathbf{C}$ OOPERATIVE relaying [1]-[3] has recently emerged as an effective technique to combat fading and exploit spatial diversity in wireless systems. Many relaying protocols have

Manuscript received April 27, 2012; revised August 18, 2012; accepted September 25, 2012. Date of publication October 10, 2012; date of current version February 12, 2013. This work was supported in part by the National Science Council (NSC), Taiwan, under Grant NSC 98-2218-E-009-008-MY3 and Grant NSC 100-2628-E-007-025-MY3. This paper was presented in part at the IEEE 11th International Symposium on Spread Spectrum Techniques and Applications, Taichung, Taiwan, October 2010, and in part at the IEEE International Conference on Communications, Kyoto, Japan, June 2011. The review of this paper was coordinated by Prof. C. Oestges.

K.-Y. Sung is with the Institute of Communications Engineering, National Chiao Tung University, Hsinchu 30010, Taiwan (e-mail: kysung@nctu.edu.tw).

Y.-W. P. Hong and C.-C. Chao are with the Institute of Communications Engineering, National Tsing Hua University, Hsinchu 30013, Taiwan (e-mail: ywhong@ee.nthu.edu.tw; ccc@ee.nthu.edu.tw).

Color versions of one or more of the figures in this paper are available online at http://ieeexplore.ieee.org.

Digital Object Identifier 10.1109/TVT.2012.2223768 been proposed in the literature [1]-[5], among which, amplifyand-forward (AF) and decode-and-forward (DF) have been the most adopted. These schemes have also been employed in conjunction with orthogonal frequency-division multiplexing (OFDM) systems in modern wireless standards, such as LongTerm Evolution-Advanced and WiMAX [6]. To fully exploit the spectral and spatial degrees of freedom, different OFDM relaying protocols have been proposed in the literature, e.g., [7][18]. Specifically, for AF OFDM(A) relay systems, power allocation and subcarrier pairing (SP) schemes have been studied in [8] and [9]-[12], respectively, and have been jointly exploited in [13] and [14]. These power allocation and SP schemes have also been studied for DF OFDM relay systems in [15], [16] and [11], [17], respectively, and a scheme that utilizes joint coding across subcarriers has also been developed in [18] as an alternative for SP. To fully exploit the available degrees of freedom in cooperative OFDM systems, knowledge of the channel state information is often needed at the receiver and/or transmitter. Without reliable channel estimation, the symbol error rate (SER) may be dominated by the channel estimation error, and the achievable cooperative diversity gains will become limited.

In this paper, we consider the use of pilot-assisted transmissions (PATs) [19], [20] to facilitate channel estimation and data symbol detection in AF OFDM relay systems. In systems employing PAT, a subset of subcarriers (called pilot tones) are dedicated to the transmission of pilot symbols, whereas others (called data tones) are utilized for data transmission. This approach has been studied extensively in the literature for pointto-point systems, e.g., [19]-[30], where the number of pilot tones, the pilot placement, and the power allocation between pilot and data symbols have been optimized to enhance system performance. In particular, pilot placement for conventional point-to-point OFDM systems has been studied in, e.g., [25][30]. With no prior information about the channel, the equalspaced pilot placement scheme has been shown to be optimal in terms of maximizing the channel capacity [25], minimizing the channel estimation error [26], [27], and minimizing SER [28]. While many of these schemes can be incorporated into cooperative relay systems [31], [32], we argue that the frequency and spatial degrees of freedom can be better exploited through the use of feedback-aided pilot transmissions.

The main contribution of this paper is to examine the use of feedback of the previous channel estimate to adaptively determine the current pilot placement among subcarriers at both source and relay in OFDM relay systems. For a given number of pilots, the placement may affect both the channel estimation error and the availability of good subcarrier channels for data transmission. To minimize channel estimation error, pilot tones should be distributed as evenly as possible across subcarriers [26], [27], whereas to increase the efficiency of 
data transmission, pilot tones should be assigned to subcarriers with bad channel conditions, leaving good subcarriers for data transmission [29], [30]. With channel estimation feedback, the optimal pilot placement scheme can be derived to exploit the tradeoff between these two effects. This is particularly important in cooperative relay systems, where channel estimation errors may have a significant impact on the SER at high signalto-noise ratio (SNR) and, thus, limit the achievable diversity gains.

In this paper, feedback-aided pilot placement schemes are proposed with the goal of maximizing the minimum effective SNR among data subcarriers. The effective SNR is defined as the receive SNR at the destination, which takes into consideration the source-to-relay (SR) and relay-to-destination (RD) channels as well as their respective channel estimation errors. The minimum effective SNR is used as the design criterion since it is what dominates the SER performance in uncoded systems [28], [30]. However, the proposed methodology can also be applied to other criteria as well (cf. Section IV). The improvement obtained by employing feedback is measured by the ratio between the minimum effective SNR achieved with the optimal feedback-aided pilot placement scheme and that achieved with the equal-spaced pilot placement scheme (i.e., the optimal scheme without feedback [25]-[28]). We refer to this ratio as the feedback gain.

In this paper, we first derive an analytic lower bound (LB) on the optimal feedback gain and obtain the asymptotic rate of increase of the feedback gain with respect to the number of subcarriers and pilot tones. Due to the complexity of finding the optimal pilot placement, the iterative pilot relocation (IterPR) scheme is proposed to efficiently and effectively determine a pilot placement with high feedback gain. In each iteration of the process, the location of the data tone with the worst effective channel gain is exchanged with the location of one of the originally selected pilot tones whenever the minimum effective SNR can be increased by doing so. This process continues iteratively until no further improvements can be obtained. We perform these studies for systems without and with SP [8], [11]. In systems without SP, each datum is forwarded by the relay on the same subcarrier it is received on. However, when SP is employed, each datum can be forwarded by the relay on a different subcarrier. The pairing between the incoming and outgoing subcarriers can be optimized for better performance. By maximizing the minimum effective SNR, the best SP scheme is shown to be an inverse mapping between the effective channel gains of the subcarriers on the SR and RD links. That is, the data received on the worst SR subcarrier are forwarded on the best RD subcarrier. Note that the optimal SP scheme obtained here is opposite to many works in the literature that consider, e.g., the maximum sum rate [8], [11] or the maximum sum effective SNR [10] as the design criterion. The effectiveness of the proposed IterPR algorithms is demonstrated through numerical simulations.

The concept of feedback-aided pilot placement has been examined for noncooperative systems in [29] and [30]. Different from these works, our studies are focused on AF OFDM relay systems, where both SR and RD channels must be estimated at the receiver. This problem is considerably more challenging than that of noncooperative systems, particularly for the case with SP. Nevertheless, we are able to obtain analytic results that show the relation between the feedback gain and the number of subcarriers and pilot tones. Our proposed IterPR scheme is also shown to achieve significant improvements over those proposed in [29] and [30]. Some preliminary results of this paper have been presented in [33] and [34]. In addition to the results in [33] and [34], this paper provides results based on more general channel models, detailed derivations of the feedback gain for cases with and without SP, and full technical proofs of the propositions and lemmas. Note that the proposed IterPR scheme can also be applied to DF systems, e.g., [11], [15]-[17], or, in particular, to joint coding systems, e.g., [18]. However, a more suitable criterion, such as the system capacity or the outage probability, should be adopted in place of the minimum effective SNR in this case. Moreover, it is necessary to remark that, with channel feedback, power allocation can also be performed among both pilot and data subcarriers. That is, given the channel estimates, the optimal power allocation can be derived by following procedures in [15] and [16] and by treating channel estimation error as an additional source of noise. However, in this paper, the pilot and data powers are fixed to focus on the effects of pilot placement.

The rest of this paper is organized as follows. In Section II, the system model and the concept of feedback-aided pilot placement are described. In Section III, analytic LBs on the optimal feedback gain are derived for cases with and without SP, and in Section IV, the IterPR pilot placement schemes are introduced for both cases. Numerical simulations and some concluding remarks are given in Sections V and VI, respectively.

Notations: In this paper, $\mathbf{A}^{T}$ and $\mathbf{A}^{H}$ represent the matrix transpose and Hermitian, respectively, and $\{\mathbf{A}\}_{i, j}$ represents the $(i, j)$ th component of the matrix $\mathbf{A} \cdot \operatorname{diag}\left(a_{1}, \ldots, a_{n}\right)$ represents a diagonal matrix with $a_{1}, \ldots, a_{n}$ as its diagonal entries, and $\mathbf{I}_{n}$ denotes the $n \times n$ identity matrix. $|\mathcal{S}|$ represents the cardinality of the set $\mathcal{S}$, and $\mathcal{S} \backslash \mathcal{T}$ represents the set subtraction of $\mathcal{T}$ from $\mathcal{S} . \mathbf{Z} \sim \mathcal{C} \mathcal{N}\left(\mathbf{0}, \boldsymbol{\Sigma}_{\mathbf{Z}}\right)$ indicates that $\mathbf{Z}$ is a zero-mean complex Gaussian random vector with covariance matrix $\boldsymbol{\Sigma}_{\mathbf{Z}}$.

\section{System Model And Problem Formulation}

Consider an AF OFDM relay system with a source, relay, and destination communicating over $N$ subcarrier channels, as shown in Fig. 1. During each OFDM symbol period, the source first transmits $N$ (pilot plus data) symbols in parallel over $N$ subcarriers (represented by the index set $\mathcal{N}=\{0,1, \ldots, N-$ $1\})$. The signals received at the relay are then amplified and forwarded to the destination. The AF relaying scheme [14] is chosen as the representative scheme here due to its wide interest in the literature and its ability to achieve full diversity through simple operations. We argue that the same design methodology can be applied to DF systems as well but will not be considered explicitly in this paper. To enable channel estimation at the receiver, pilots are inserted into a subset of subcarriers, called pilot tones, at both the source and the relay. The pilot tones adopted by the source and relay may be different and are indicated by the index sets $\mathcal{P}_{S} \subset \mathcal{N}$ and $\mathcal{P}_{R} \subset \mathcal{N}$, respectively. The sets of data subcarriers adopted by the source and relay are then represented by the index sets $\mathcal{D}_{S}=\mathcal{N} \backslash \mathcal{P}_{S}$ and $\mathcal{D}_{R}=$ $\mathcal{N} \backslash \mathcal{P}_{R}$, respectively. Suppose that the number of pilot tones 


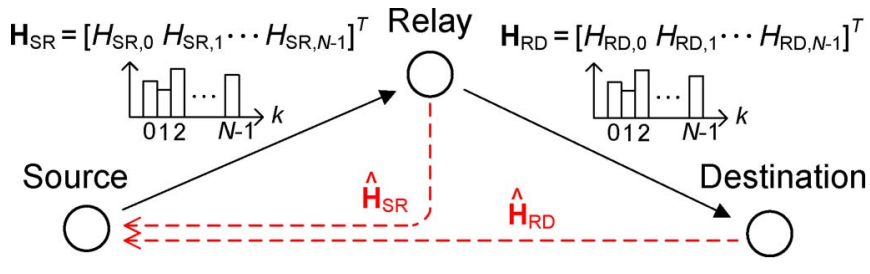

Fig. 1. OFDM relay system model with channel feedback.

is $\left|\mathcal{P}_{S}\right|=\left|\mathcal{P}_{R}\right|=N_{p}=\alpha N$, where $\alpha$ is the proportion of subcarriers used as pilot tones, and the number of data tones is $\left|\mathcal{D}_{S}\right|=\left|\mathcal{D}_{R}\right|=N_{d}=N-N_{p}$. When SP is employed, the relay must determine on which subcarrier each received data is to be forwarded. This operation is determined by the SP function $\varphi: \mathcal{D}_{S} \rightarrow \mathcal{D}_{R}$, which is a one-to-one function with $\varphi(k)$ representing the index of the subcarrier used to forward the data received on the $k$ th subcarrier. This method has often been adopted in the literature, e.g. [9], [10], [12], [35], and [36], to better exploit diversity in the frequency domain. In the case without SP, the function is defined by $\varphi(k)=k$ for all $k \in \mathcal{D}_{S}=\mathcal{D}_{R}$.

Suppose that the SR and RD links are modeled as multipath channels with $L_{1}$ and $L_{2}$ independent fading paths and that $\mathbf{h}_{\mathrm{SR}}=\left[h_{\mathrm{SR}, 0}, \ldots, h_{\mathrm{SR}, L_{1}-1}\right]^{T}$ and $\mathbf{h}_{\mathrm{RD}}=\left[h_{\mathrm{RD}, 0}, \ldots\right.$, $\left.h_{\mathrm{RD}, L_{2}-1}\right]^{T}$ are their respective discrete-time channel impulse responses. Both $L_{1}$ and $L_{2}$ are assumed to be less than the guard interval so that intersymbol interference can be eliminated. Each channel tap is modeled as an independent complex Gaussian random variable, i.e., $\mathbf{h}_{\mathrm{SR}} \sim \mathcal{C N}\left(\mathbf{0}, \boldsymbol{\Sigma}_{\mathrm{SR}}\right)$ and $\mathbf{h}_{\mathrm{RD}} \sim \mathcal{C N}\left(\mathbf{0}, \boldsymbol{\Sigma}_{\mathrm{RD}}\right)$, where $\mathbf{0}$ is an all-zero vector, $\boldsymbol{\Sigma}_{\mathrm{SR}}=$ $\mathrm{E}\left[\mathbf{h}_{\mathrm{SR}} \mathbf{h}_{\mathrm{SR}}^{H}\right]=\operatorname{diag}\left(\sigma_{\mathrm{SR}, 0}^{2}, \ldots, \sigma_{\mathrm{SR}, L_{1}-1}^{2}\right), \quad$ and $\quad \boldsymbol{\Sigma}_{\mathrm{RD}}=$ $\mathrm{E}\left[\mathbf{h}_{\mathrm{RD}} \mathbf{h}_{\mathrm{RD}}^{H}\right]=\operatorname{diag}\left(\sigma_{\mathrm{RD}, 0}^{2}, \ldots, \sigma_{\mathrm{RD}, L_{2}-1}^{2}\right)$. It is assumed that $\mathrm{E}\left[\left\|\mathbf{h}_{\mathrm{SR}}\right\|^{2}\right]=\sum_{\ell=0}^{L_{1}-1} \sigma_{\mathrm{SR}, \ell}^{2}=\sigma_{\mathrm{SR}}^{2}$ and $\mathrm{E}\left[\left\|\mathbf{h}_{\mathrm{RD}}\right\|^{2}\right]=$ $\sum_{\ell=0}^{L_{2}-1} \sigma_{\mathrm{RD}, \ell}^{2}=\sigma_{\mathrm{RD}}^{2}$, where $\sigma_{\mathrm{SR}}^{2}$ and $\sigma_{\mathrm{RD}}^{2}$ can be used to model the effect of path loss on the SR and RD links, respectively. The frequency-domain channel vectors are given by $\mathbf{H}_{\mathrm{SR}}=\left[H_{\mathrm{SR}, 0}, \ldots, H_{\mathrm{SR}, N-1}\right]^{T}=\sqrt{N} \mathbf{W}_{L_{1}} \mathbf{h}_{\mathrm{SR}}$ and $\mathbf{H}_{\mathrm{RD}}=$ $\left[H_{\mathrm{RD}, 0}, \ldots, H_{\mathrm{RD}, N-1}\right]^{T}=\sqrt{N} \mathbf{W}_{L_{2}} \mathbf{h}_{\mathrm{RD}}$, where $\mathbf{W}_{L}$ is defined as the truncated unit-norm fast Fourier transform matrix of size $N \times L$ with $\{\mathbf{W}\}_{k, \ell}=1 / \sqrt{N} \exp (-j 2 \pi \times$ $(k-1)(\ell-1) / N)$.

Let $s_{k}$ be the symbol transmitted by the source on the $k$ th subcarrier, and assume that the pilot symbols have constant power with $\left|s_{k}\right|^{2}=\rho_{p}, \forall k \in \mathcal{P}_{S}$, and that data symbols on different subcarriers have the same average power with $\mathrm{E}\left[s_{k}^{2}\right]=$ $\rho_{d}, \forall k \in \mathcal{D}_{S}$. The signal received at the relay over the $N$ subcarriers is given by

$$
\mathbf{Y}_{R}=\left[Y_{\mathrm{R}, 0}, \ldots, Y_{\mathrm{R}, N-1}\right]^{T}=\mathbf{S H}_{\mathrm{SR}}+\mathbf{Z}_{R}
$$

where $\mathbf{S}=\operatorname{diag}\left(s_{0}, s_{1}, \ldots, s_{N-1}\right)$, and $\mathbf{Z}_{R} \sim \mathcal{C N}\left(\mathbf{0}, \sigma_{z}^{2} \mathbf{I}_{N}\right)$ is the additive white Gaussian noise (AWGN) vector. By extracting the entries corresponding to the index sets $\mathcal{P}_{S}$ and $\mathcal{D}_{S}$, the received vector $\mathbf{Y}_{R}$ can be further divided into two vectors $\mathbf{Y}_{R}^{(p)}$ and $\mathbf{Y}_{R}^{(d)}$, respectively. The relay can use the received pilot signals $\mathbf{Y}_{R}^{(p)}$ to obtain an estimate of $\mathbf{H}_{\mathrm{SR}}$ and send this estimate to the destination through a feedforward control channel [37].
Upon receiving the signal in (1), the relay will amplify and forward the signals received on the data subcarriers while transmitting newly generated symbols on the pilot subcarriers to facilitate estimation of the RD channel vector $\mathbf{H}_{\mathrm{RD}}$ at the destination [37]. Note that the pilot symbols transmitted by the source are used to estimate the SR channel $\mathbf{H}_{\mathrm{SR}}$ at the relay, and those transmitted by the relay are used to estimate the RD channel vector $\mathbf{H}_{\mathrm{RD}}$ at the destination, ${ }^{1}$ similar to that considered in [37]. Specifically, let $\tilde{s}_{k}$ be the signal transmitted by the relay on the $k$ th subcarrier. In this case, $\tilde{s}_{k}$ will be equal to a newly generated pilot symbol with $\left|\tilde{s}_{k}\right|^{2}=\rho_{p}$ for $k \in \mathcal{P}_{R}$ and will be equal to the amplified signal $\Lambda_{k} Y_{\mathrm{R}, \varphi^{-1}(k)}$ for $k \in \mathcal{D}_{R}$, where $\varphi^{-1}$ is the inverse function of $\varphi$, and $\Lambda_{k}=$ $\sqrt{\rho_{d} /\left(\rho_{d}\left|H_{\mathrm{SR}, \varphi^{-1}(k)}\right|^{2}+\sigma_{z}^{2}\right)}$ is the amplification factor chosen such that the received power per data subcarrier at the relay is scaled to $\rho_{d}$. Let $\boldsymbol{\Phi}$ be an $N_{d} \times N_{d}$ matrix that is obtained by taking the $N_{d}$ nonzero rows and columns of an $N \times N$ matrix with 1's in the $(\varphi(k), k)$ th entry for all $k \in \mathcal{D}_{S}$ and 0 's everywhere else. Hence, by premultiplying the received data signals $\mathbf{Y}_{R}^{(d)}$ with $\boldsymbol{\Phi}$, the signals transmitted on the data subcarriers on the SR link are relocated to their corresponding data subcarriers on the RD link. The signals received on the pilot and data subcarriers at the destination can then be written as vectors

$$
\begin{aligned}
\mathbf{Y}_{\mathrm{D}}^{(p)} & =\tilde{\mathbf{S}}^{(p)} \mathbf{H}_{\mathrm{RD}}^{(p)}+\mathbf{Z}_{\mathrm{D}}^{(p)} \\
\mathbf{Y}_{\mathrm{D}}^{(d)} & =\boldsymbol{\Lambda}^{(d)} \operatorname{diag}\left(\mathbf{\Phi} \mathbf{Y}_{R}^{(d)}\right) \mathbf{H}_{\mathrm{RD}}^{(d)}+\mathbf{Z}_{\mathrm{D}}^{(d)}
\end{aligned}
$$

where $\tilde{\mathbf{S}}^{(p)}$ is a diagonal matrix containing on its diagonal the $N_{p}$ newly generated pilot symbols at the relay, $\boldsymbol{\Lambda}^{(d)}$ is a diagonal matrix with the amplification factor $\Lambda_{k}$ for $k \in \mathcal{D}_{R}$ on its diagonal, $\mathbf{H}_{\mathrm{RD}}^{(p)}$ and $\mathbf{H}_{\mathrm{RD}}^{(d)}$ are the $\mathrm{RD}$ channel coefficients on the pilot and data subcarriers, respectively, and $\mathbf{Z}_{\mathrm{D}}^{(p)} \sim$ $\mathcal{C N}\left(\mathbf{0}, \sigma_{z}^{2} \mathbf{I}_{N_{p}}\right)$ and $\mathbf{Z}_{\mathrm{D}}^{(d)} \sim \mathcal{C N}\left(\mathbf{0}, \sigma_{z}^{2} \mathbf{I}_{N_{d}}\right)$ are the AWGNs.

Here, we assume that the minimum mean square error (MMSE) estimator [38] is used to compute the channel estimates at both the relay and the destination. Given the pilot placement, the MMSE estimate of the SR channel vector $\mathbf{H}_{\mathrm{SR}}$ can be computed as

$$
\begin{aligned}
\hat{\mathbf{H}}_{\mathrm{SR}} & =\mathrm{E}\left[\mathbf{H}_{\mathrm{SR}}\left(\mathbf{Y}_{R}^{(p)}\right)^{H}\right]\left(\mathrm{E}\left[\mathbf{Y}_{R}^{(p)}\left(\mathbf{Y}_{R}^{(p)}\right)^{H}\right]\right)^{-1} \mathbf{Y}_{R}^{(p)} \\
& =N \mathbf{W}_{L_{1}} \boldsymbol{\Sigma}_{\mathrm{SR}}\left(\mathbf{W}_{L_{1}}^{(p)}\right)^{H} \mathbf{Q}_{\mathrm{SR}}^{-1}\left(\mathbf{S}^{(p)}\right)^{H} \mathbf{Y}_{R}^{(p)}
\end{aligned}
$$

where $\mathbf{W}_{L_{1}}^{(p)}$ is an $N_{p} \times L_{1}$ matrix formed by the rows of $\mathbf{W}_{L_{1}}$ that correspond to the pilot indices in $\mathcal{P}_{S}, \boldsymbol{\Sigma}_{\mathrm{SR}}$ is

\footnotetext{
${ }^{1}$ Some works in the literature on AF relaying consider the estimation of the combined SR and RD channel, as opposed to estimating them separately, as done in this work. However, the approach considered here has several advantages. In particular, knowledge of both the SR and the RD channels allows for the use of more advanced relaying schemes such as the subcarrier pairing considered in this paper. Several conventional schemes, such as the variable-gain $\mathrm{AF}$ relaying scheme [2], also require separate knowledge of the SR channel at the relay. Moreover, optimal detection at the destination also requires separate knowledge of both channels since the effective noise variance depends on the RD channel. Knowledge of only the combined source-relaydestination channel only allows for the use of suboptimal schemes such as the minimum distance detector.
} 
the covariance matrix of the time-domain vector $\mathbf{h}_{\mathrm{SR}}$, and $\mathbf{Q}_{\mathrm{SR}}=N \rho_{p} \mathbf{W}_{L_{1}}^{(p)} \boldsymbol{\Sigma}_{\mathrm{SR}}\left(\mathbf{W}_{L_{1}}^{(p)}\right)^{H}+\sigma_{z}^{2} \mathbf{I}_{N_{p}} . \quad$ Similarly, the MMSE estimate of $\mathbf{H}_{\mathrm{RD}}$ can be computed as

$$
\hat{\mathbf{H}}_{\mathrm{RD}}=N \mathbf{W}_{L_{2}} \boldsymbol{\Sigma}_{\mathrm{RD}}\left(\mathbf{W}_{L_{2}}^{(p)}\right)^{H} \mathbf{Q}_{\mathrm{RD}}^{-1}\left(\tilde{\mathbf{S}}^{(p)}\right)^{H} \mathbf{Y}_{\mathrm{D}}^{(p)}
$$

where $\mathbf{W}_{L_{2}}^{(p)}$ is an $N_{p} \times L_{2}$ matrix formed by the rows of $\mathbf{W}_{L_{2}}$ that correspond to the pilot indices in $\mathcal{P}_{R}, \boldsymbol{\Sigma}_{\mathrm{RD}}$ is the covariance matrix of $\mathbf{h}_{\mathrm{RD}}$, and $\mathbf{Q}_{\mathrm{RD}}=N \rho_{p} \mathbf{W}_{L_{2}}^{(p)} \boldsymbol{\Sigma}_{\mathrm{RD}}\left(\mathbf{W}_{L_{2}}^{(p)}\right)^{H}+$ $\sigma_{z}^{2} \mathbf{I}_{N_{p}}$. In this case, the channel estimation errors $\tilde{\mathbf{H}}_{\mathrm{SR}}=$ $\mathbf{H}_{\mathrm{SR}}-\hat{\mathbf{H}}_{\mathrm{SR}}$ and $\tilde{\mathbf{H}}_{\mathrm{RD}}=\mathbf{H}_{\mathrm{RD}}-\hat{\mathbf{H}}_{\mathrm{RD}}$ can be shown to be zero mean with covariance matrices

$$
\begin{aligned}
\mathrm{E} & {\left[\tilde{\mathbf{H}}_{\mathrm{SR}}\left(\tilde{\mathbf{H}}_{\mathrm{SR}}\right)^{H}\right] } \\
& =N \mathbf{W}_{L_{1}}\left(\boldsymbol{\Sigma}_{\mathrm{SR}}^{-1}+\gamma_{p} N\left(\mathbf{W}_{L_{1}}^{(p)}\right)^{H} \mathbf{W}_{L_{1}}^{(p)}\right)^{-1}\left(\mathbf{W}_{L_{1}}\right)^{H} \\
\mathrm{E} & {\left[\tilde{\mathbf{H}}_{\mathrm{RD}}\left(\tilde{\mathbf{H}}_{\mathrm{RD}}\right)^{H}\right] } \\
& =N \mathbf{W}_{L_{2}}\left(\boldsymbol{\Sigma}_{\mathrm{RD}}^{-1}+\gamma_{p} N\left(\mathbf{W}_{L_{2}}^{(p)}\right)^{H} \mathbf{W}_{L_{2}}^{(p)}\right)^{-1}\left(\mathbf{W}_{L_{2}}\right)^{H}
\end{aligned}
$$

where $\gamma_{p}=\rho_{p} / \sigma_{z}^{2}$ is the transmit SNR of the pilot signal. The $k$ th diagonal elements of $\mathrm{E}\left[\tilde{\mathbf{H}}_{\mathrm{SR}}\left(\tilde{\mathbf{H}}_{\mathrm{SR}}\right)^{H}\right]$ and $\mathrm{E}\left[\tilde{\mathbf{H}}_{\mathrm{RD}}\left(\tilde{\mathbf{H}}_{\mathrm{RD}}\right)^{H}\right]$ are the estimation error variances of $\hat{H}_{\mathrm{SR}, k}$ and $\hat{H}_{\mathrm{RD}, k}$ and are denoted by $\epsilon_{\mathrm{SR}, k}$ and $\epsilon_{\mathrm{RD}, k}$, respectively. Notice that, by using the expressions in (6) and (7), the estimation error variances can be computed for any given pilot placement $\mathcal{P}_{S}$ and $\mathcal{P}_{R}$.

With the channel estimates $\hat{\mathbf{H}}_{\mathrm{SR}}, \hat{\mathbf{H}}_{\mathrm{RD}}$ and the channel estimation errors $\tilde{\mathbf{H}}_{\mathrm{SR}}, \tilde{\mathbf{H}}_{\mathrm{RD}}$, the signals received on the data subcarriers at the destination can be rewritten as

$$
\begin{aligned}
& \mathbf{Y}_{\mathrm{D}}^{(d)}=\mathbf{\Lambda}^{(d)} \operatorname{diag}\left(\boldsymbol{\Phi} \mathbf{S}^{(d)}\right) \operatorname{diag}\left(\boldsymbol{\Phi} \hat{\mathbf{H}}_{\mathrm{SR}}^{(d)}\right) \hat{\mathbf{H}}_{\mathrm{RD}}^{(d)} \\
& +\underbrace{\boldsymbol{\Lambda}^{(d)} \operatorname{diag}\left(\boldsymbol{\Phi} \mathbf{S}^{(d)}\right)\left(\operatorname{diag}\left(\boldsymbol{\Phi} \hat{\mathbf{H}}_{\mathrm{SR}}^{(d)}\right) \tilde{\mathbf{H}}_{\mathrm{RD}}^{(d)}+\operatorname{diag}\left(\boldsymbol{\Phi} \tilde{\mathbf{H}}_{\mathrm{SR}}^{(d)}\right) \mathbf{H}_{\mathrm{RD}}^{(d)}\right)}
\end{aligned}
$$

Noise contributed by channel estimation errors

$$
+\underbrace{\Lambda^{(d)} \operatorname{diag}\left(\mathbf{H}_{\mathrm{RD}}^{(d)}\right) \boldsymbol{\Phi} \mathbf{Z}_{R}^{(d)}+\mathbf{Z}_{\mathrm{D}}^{(d)}}
$$

Additive noise at relay and destination

where $\hat{\mathbf{H}}_{\mathrm{SR}}^{(d)}$ and $\hat{\mathbf{H}}_{\mathrm{RD}}^{(d)}$ are the estimated SR and RD channels on the corresponding data subcarriers. Notice that the data transmitted by the source on subcarrier $k$ is received by the destination on subcarrier $\varphi(k)$. Since $\hat{\mathbf{H}}_{\mathrm{SR}}, \tilde{\mathbf{H}}_{\mathrm{SR}}, \hat{\mathbf{H}}_{\mathrm{RD}}$, and $\tilde{\mathbf{H}}_{\mathrm{RD}}$ are mutually uncorrelated, the effective SNR corresponding to the signal transmitted by the source on subcarrier $k$ is

\begin{tabular}{|c|c|}
\hline & number of pilot, data, and to \\
\hline $\mathcal{P}_{\mathrm{S}}, \mathcal{P}_{\mathrm{R}}$ & $\begin{array}{l}\text { index sets of the pilot subcarriers adopted by the source } \\
\text { and the relay, respectively }\end{array}$ \\
\hline $\mathcal{D}_{\mathrm{S}}, \mathcal{D}_{\mathrm{R}}$ & $\begin{array}{l}\text { index sets of the data subcarriers adopted by the source } \\
\text { and the relay, respectively }\end{array}$ \\
\hline $\begin{array}{l}\mathcal{N} \\
\varphi(\cdot)\end{array}$ & $\begin{array}{l}\text { index set of total subcarriers; } \mathcal{N}=\mathcal{P}_{\mathrm{S}} \cup \mathcal{D}_{\mathrm{S}}=\mathcal{P}_{\mathrm{R}} \cup \mathcal{D}_{\mathrm{R}} \\
\text { subcarrier paring function; a one-to-one mapping from } \mathcal{D}_{\mathrm{S}} \\
\text { to } \mathcal{D}_{\mathrm{R}}\end{array}$ \\
\hline$\Phi$ & $\begin{array}{l}\text { permutation matrix generated by the subcarrier pairing } \\
\text { function } \varphi(\cdot)\end{array}$ \\
\hline $\mathbf{S}$ & $\begin{array}{l}\text { transmitted signal matrix containing on its diagonal the } \\
\text { data on all subcarriers }\end{array}$ \\
\hline $\mathbf{S}^{(p)}\left(\mathbf{S}^{(d)}\right)$ & $\begin{array}{l}\text { transmitted pilot (data) matrix containing on its diagonal } \\
\text { the pilot (data) symbols }\end{array}$ \\
\hline$\tilde{\mathbf{S}}^{(p)}$ & $\begin{array}{l}\text { retransmitted pilot matrix at the relay containing on its } \\
\text { diagonal the regenerated pilots }\end{array}$ \\
\hline $\mathbf{H}_{\mathrm{SR}}, \mathbf{H}_{\mathrm{RD}}$ & $\begin{array}{l}\text { frequency response vectors of the SR and RD channels, } \\
\text { respectively }\end{array}$ \\
\hline$\hat{\mathbf{H}}_{\mathrm{SR}}, \hat{\mathbf{H}}_{\mathrm{RD}}$ & $\begin{array}{l}\text { estimated channel response vectors of the SR and RD } \\
\text { channels, respectively }\end{array}$ \\
\hline$\tilde{\mathbf{H}}_{\mathrm{SR}}, \tilde{\mathbf{H}}_{\mathrm{RD}}$ & $\begin{array}{l}\text { channel estimation error variance vectors of the SR and } \\
\text { RD channels, respectively }\end{array}$ \\
\hline $\mathbf{H}_{\mathrm{SR}}^{(p)}, \mathbf{H}_{\mathrm{RD}}^{(p)}$ & $\begin{array}{l}\text { frequency response vectors on the pilot subcarriers for the } \\
\text { SR and RD channels, respectively }\end{array}$ \\
\hline $\mathbf{H}_{\mathrm{SR}}^{(d)}, \mathbf{H}_{\mathrm{RD}}^{(d)}$ & $\begin{array}{l}\text { frequency response vectors on the data subcarriers for the } \\
\text { SR and RD channels, respectively }\end{array}$ \\
\hline & received signal vector at the relay \\
\hline $\mathbf{Y}_{\mathrm{R}}^{(p)}, \mathbf{Y}_{\mathrm{D}}^{(p)}$ & $\begin{array}{l}\text { received signal vectors on the pilot subcarriers at the relay } \\
\text { and the destination, respectively }\end{array}$ \\
\hline $\mathbf{Y}_{\mathrm{R}}^{(d)}, \mathbf{Y}_{\mathrm{D}}^{(d)}$ & $\begin{array}{l}\text { received signal vectors on the data subcarriers at the relay } \\
\text { and the destination, respectively }\end{array}$ \\
\hline $\mathbf{Z}_{\mathrm{R}}, \mathbf{Z}_{\mathrm{D}}$ & $\begin{array}{l}\text { additive noise vectors at the relay and the destination, } \\
\text { respectively }\end{array}$ \\
\hline $\mathbf{Z}_{\mathrm{R}}^{(p)}, \mathbf{Z}_{\mathrm{D}}^{(p)}$ & $\begin{array}{l}\text { additive noise vector on the pilot subcarriers at the relay } \\
\text { and the destination, respectively }\end{array}$ \\
\hline $\mathbf{Z}_{\mathrm{R}}^{(d)}, \mathbf{Z}_{\mathrm{D}}^{(d)}$ & $\begin{array}{l}\text { additive noise vector on the data subcarriers at the relay } \\
\text { and the destination, respectively }\end{array}$ \\
\hline $\boldsymbol{\Sigma}_{\mathrm{SR}}, \boldsymbol{\Sigma}_{\mathrm{RD}}$ & $\begin{array}{l}\text { channel correlation matrices containing on its diagonal the } \\
\text { variance of each channel tap }\end{array}$ \\
\hline & transmit power of the pilot and data symbols, respectively \\
\hline $\begin{array}{l}\gamma_{p}, \gamma_{d} \\
\sigma_{z}^{2}\end{array}$ & $\begin{array}{l}\text { transmit SNR of the pilot and data symbols } \\
\text { noise variance }\end{array}$ \\
\hline$\xi_{k}^{2}$ & effective SNR on subcarrier $k$ \\
\hline
\end{tabular}
(cf. Appendix A)

$$
\xi_{k}=\gamma_{d} \cdot \frac{\left|\hat{H}_{\mathrm{SR}, k}\right|^{2} \cdot\left|\hat{H}_{\mathrm{RD}, \varphi(k)}\right|^{2}}{\left|\hat{H}_{\mathrm{SR}, k}\right|^{2}+\left|\hat{H}_{\mathrm{RD}, \varphi(k)}\right|^{2}+\zeta_{k}+1 / \gamma_{d}}
$$

where $\gamma_{d}=\rho_{d} / \sigma_{z}^{2}$ is the transmit SNR of the data symbol (i.e., the ratio between the transmit power of the data symbol and the
TABLE I

SUMMARY OF VARIABLES

noise variance), and

$$
\begin{aligned}
\zeta_{k}=\gamma_{d} \cdot\left(\left|\hat{H}_{\mathrm{SR}, k}\right|^{2} \epsilon_{\mathrm{RD}, \varphi(k)}+\left|\hat{H}_{\mathrm{RD}, \varphi(k)}\right|^{2} \epsilon_{\mathrm{SR}, k}\right. \\
\left.+\epsilon_{\mathrm{SR}, k} \epsilon_{\mathrm{RD}, \varphi(k)}\right)+\epsilon_{\mathrm{SR}, k}+\epsilon_{\mathrm{RD}, \varphi(k)}
\end{aligned}
$$

can be viewed as the effective estimation error on the combined source-relay-destination (SRD) channel. Notice that the effective SNR is affected by the choice of pilot and data subcarriers, particularly through the channel gains of the selected data subcarriers and the estimation error. All the previously used variables are summarized in Table I.

Let us consider the minimum effective SNR, i.e., $\min \xi_{k}$, as the design criterion since it is what eventually dominates the SER of uncoded systems at high SNR. In this case, the optimal sets of data subcarriers at the source and relay (i.e., $\mathcal{D}_{S}^{\star}$ and $\mathcal{D}_{R}^{\star}$ ) and the optimal SP function (i.e., $\varphi^{\star}$ ) can be found by maximizing the minimum effective SNR, i.e.,

$$
\left(\varphi^{\star}, \mathcal{D}_{S}^{\star}, \mathcal{D}_{R}^{\star}\right)=\underset{\varphi, \mathcal{D}_{S}, \mathcal{D}_{R}}{\arg \max }\left\{\min _{k \in \mathcal{D}_{S}} \xi_{k}\right\} .
$$


The optimal pilot locations are, thus, given by

$$
\mathcal{P}_{S}^{\star}=\mathcal{N} \backslash \mathcal{D}_{S}^{\star} \quad \text { and } \quad \mathcal{P}_{R}^{\star}=\mathcal{N} \backslash \mathcal{D}_{R}^{\star} .
$$

Notice that, in (10), the effective SNRs must be computed for all possible pilot placements. However, these values depend on the channel estimates computed under each pilot placement, which cannot be obtained without actually transmitting the pilot symbols. In practice, we propose to approximate the channel estimates with those obtained through feedback in the previous time frame. We argue that these approximations will be sufficiently accurate if the time between subsequent channel estimates is smaller than the coherence time and if the feedback channel is sufficiently reliable. The effect of approximation errors will be examined through simulations in Section V but will not be considered explicitly in our analysis.

Notice that the problem in (10) requires a search over all possible combinations of $\left(\mathcal{D}_{S}, \mathcal{D}_{R}\right)$ (or, equivalently, $\left(\mathcal{P}_{S}, \mathcal{P}_{R}\right)$ ) and SP functions $\varphi$. Unfortunately, an efficient solution for such a problem does not yet exist, even for the case without relays [29], [30]. Therefore, even if the optimal SP function can be found explicitly (which is the case here, as shown in Section III-B), an exhaustive search over all possible choices of $\left(\mathcal{D}_{S}, \mathcal{D}_{R}\right)$ still requires complexity of $\left(\begin{array}{c}N \\ N_{d}\end{array}\right)^{2}$, which rapidly becomes intractable as $N$ increases. Hence, in the following section, we derive theoretical LBs on the optimal performance to gather insight on the gains achievable with the feedback-aided scheme and further propose, in Section IV, low-complexity algorithms that can effectively determine pilot placements with high feedback gain.

Remark 1: In practice, the proposed feedback-aided scheme can be realized by having the destination compute the optimal pilot placement and SP based on its knowledge of the SR and RD channels and transmit the selection to both the source and relay. The SR and RD channels are required at the destination to perform coherent detection in any case, and thus, no extra overhead is needed to acquire this information. However, additional overhead is required to feedback the selected pilot placement and SP. For systems without SP, the number of possible combinations of pilot locations is $\left(\begin{array}{c}N \\ N_{p}\end{array}\right)$, and thus, $\log _{2}\left(\begin{array}{c}N \\ N_{p}\end{array}\right)$ bits are required to feedback the selected pilot placement to the source and relay. For systems with SP, pilot locations can be different at the source and relay, and thus, $\log _{2}\left(\begin{array}{c}N \\ N_{p}\end{array}\right)^{2}$ number of bits is required to feedback the selected pilot placement. The SP, however, requires another $\log _{2}\left(N-N_{p}\right)$ ! bits. Therefore, a total of $\log _{2}\left(\left(\begin{array}{c}N \\ N_{p}\end{array}\right)^{2} \cdot\left(N-N_{p}\right)\right)$ bits are required for feedback in systems with SP. Note that the pilot placement need not be updated every symbol period, and thus, the update frequency can be adjusted according to the overhead affordable by the system.

\section{Analysis of Achievable Feedback Gain}

In this section, we analyze the feedback gain attainable with the optimal feedback-aided pilot placement scheme as compared to the equal-spaced pilot placement scheme [25], [26], [28]. Note that the equal-spaced and equal-powered pilot placement scheme is optimal under the MMSE criterion when no channel feedback is available (cf. [26] and [27]).
Specifically, let us define the feedback gain as

$$
\mathcal{G}^{\mathrm{FB}}=\frac{\mathrm{E}\left[\xi_{(\mathrm{min})}^{\mathrm{FB}}\right]}{\mathrm{E}\left[\xi_{(\text {min })}^{\mathrm{EQ}}\right]}
$$

where $\xi_{(\min )}^{\mathrm{FB}}$ is the minimum effective SNR achieved with the optimal feedback-aided pilot placement scheme along with the optimal SP, and $\xi_{(\min )}^{\mathrm{EQ}}$ is that under the equal-spaced pilot placement scheme (also with optimal SP). Here, we consider the high SNR scenario on both training and data transmission, where $1 / \gamma_{d} \ll\left|\hat{H}_{\mathrm{SR}, k}\right|^{2},\left|\hat{H}_{\mathrm{RD}, \varphi(k)}\right|^{2}$ and $\epsilon_{\mathrm{SR}, k}, \epsilon_{\mathrm{RD}, \varphi(k)} \ll$ $\left|\hat{H}_{\mathrm{SR}, k}\right|^{2},\left|\hat{H}_{\mathrm{RD}, \varphi(k)}\right|^{2}$; therefore, the terms $\epsilon_{\mathrm{SR}, k} \epsilon_{\mathrm{RD}, \varphi(k)}$ in (9) can be neglected. The denominator in (8) can then be approximated as

$$
\begin{aligned}
& \left|\hat{H}_{\mathrm{SR}, k}\right|^{2}+\left|\hat{H}_{\mathrm{RD}, \varphi(k)}\right|^{2}+\zeta_{k}+1 / \gamma_{d} \\
& \quad \approx\left|\hat{H}_{\mathrm{SR}, k}\right|^{2}\left(1+\gamma_{d} \epsilon_{\mathrm{RD}, \varphi(k)}\right)+\left|\hat{H}_{\mathrm{RD}, \varphi(k)}\right|^{2}\left(1+\gamma_{d} \epsilon_{\mathrm{SR}, k}\right) .
\end{aligned}
$$

Consequently, the effective SNR can be approximated as

$$
\xi_{k} \simeq \gamma_{d} \cdot \frac{1}{\left(\frac{1+\gamma_{d} \epsilon_{\mathrm{SR}, k}}{\left|\hat{H}_{\mathrm{SR}, k}\right|^{2}}\right)+\left(\frac{1+\gamma_{d} \epsilon_{\mathrm{RD}, \varphi(k)}}{\left|\hat{H}_{\mathrm{RD}, \varphi(k)}\right|^{2}}\right)}
$$

which is basically the multiplication of the transmit SNR, i.e., $\gamma_{d}$, and one half the harmonic mean of the equivalent channel gains $\left|\hat{H}_{\mathrm{SR}, k}\right|^{2} /\left(1+\gamma_{d} \epsilon_{\mathrm{SR}, k}\right)$ and $\left|\hat{H}_{\mathrm{RD}, \varphi(k)}\right|^{2} /(1+$ $\left.\gamma_{d} \epsilon_{\mathrm{RD}, \varphi(k)}\right)$. Notice that the equivalent channel gains $\left|\hat{H}_{\mathrm{SR}, k}\right|^{2} /\left(1+\gamma_{d} \epsilon_{\mathrm{SR}, k}\right)$ and $\left|\hat{H}_{\mathrm{RD}, \varphi(k)}\right|^{2} /\left(1+\gamma_{d} \epsilon_{\mathrm{RD}, \varphi(k)}\right)$ can be computed by the source for all $k \in \mathcal{D}_{S}$ and $\varphi(k) \in \mathcal{D}_{R}$ once the pilot subcarriers at the source and relay (i.e., the sets $\mathcal{P}_{S}$ and $\mathcal{P}_{R}$ ) have been determined.

In the equal-spaced pilot placement scheme, the sets of pilot and data subcarriers are given by $\mathcal{P}_{S}=\mathcal{P}_{R}=\mathcal{P}^{\mathrm{EQ}} \triangleq$ $\left\{i\left\lfloor N / N_{p}\right\rfloor: i=0, \ldots, N_{p}-1\right\} \quad$ and $\quad \mathcal{D}_{S}=\mathcal{D}_{R}=\mathcal{D}^{\mathrm{EQ}} \triangleq$ $\mathcal{N} \backslash \mathcal{P}^{\mathrm{EQ}}$, respectively. ${ }^{2}$ In this case, the channel estimation errors given in (6) and (7) are

$\epsilon_{\mathrm{SR}, k}=\sum_{\ell=0}^{L_{1}-1} \frac{1}{\frac{1}{\sigma_{\mathrm{SR}, \ell}^{2}}+N_{p} \gamma_{p}}$ and $\epsilon_{\mathrm{RD}, k}=\sum_{\ell=0}^{L_{2}-1} \frac{1}{\frac{1}{\sigma_{\mathrm{RD}, \ell}^{2}}+N_{p} \gamma_{p}}$

for all $k \in \mathcal{D}^{\mathrm{EQ}}$. Note that the equal-spaced pilot placement scheme yields the same error variance for any offset in its placement. By defining $\varepsilon_{1}=1+\gamma_{d} \cdot \sum_{\ell=0}^{L_{1}-1} 1 /\left(1 / \sigma_{\mathrm{SR}, \ell}^{2}+N_{p} \gamma_{p}\right)$ and $\varepsilon_{2}=1+\gamma_{d} \cdot \sum_{\ell=0}^{L_{2}-1} 1 /\left(1 / \sigma_{\mathrm{RD}, \ell}^{2}+N_{p} \gamma_{p}\right)$, the minimum effective SNR for the equal-spaced pilot placement can be expressed as

$$
\xi_{(\min )}^{\mathrm{EQ}}=\max _{\varphi} \min _{k \in \mathcal{D}^{\mathrm{EQ}}} \gamma_{d} \cdot \frac{1}{\left(\frac{\varepsilon_{1}}{\left|\hat{H}_{\mathrm{SR}, k}\right|^{2}}\right)+\left(\frac{\varepsilon_{2}}{\left|\hat{H}_{\mathrm{RD}, \varphi(k)}\right|^{2}}\right)} .
$$

For the feedback-aided pilot placement scheme, the optimal sets of pilot subcarriers $\mathcal{P}_{S}^{\star}$ and $\mathcal{P}_{R}^{\star}$ are determined by

\footnotetext{
${ }^{2}$ Notice that the pilot subcarrier indices in the equal-spaced pilot placement scheme can be expressed more generally as $\mathcal{P}_{S}=\mathcal{P}_{R}=\mathcal{P}^{\mathrm{EQ}}=$ $\left\{i_{0}+i\left\lfloor N / N_{p}\right\rfloor: i=0, \ldots, N_{p}-1\right\}$ for any integer $0 \leq i_{0} \leq\left\lfloor N / N_{p}\right\rfloor-$ 1. However, it can be shown that the error covariance matrices in (6) and (7) do not change with $i_{0}$. Hence, we simply set $i_{0}=0$ in this work.
} 
maximizing the minimum effective SNR, as shown in (10) and (11). However, due to the complexity of finding $\mathcal{P}_{S}^{\star}$ and $\mathcal{P}_{R}^{\star}$, the minimum effective SNR obtained under the optimal feedbackaided policy is difficult to evaluate. Hence, we instead examine an LB that is derived by analyzing a suboptimal but tractable feedback-aided pilot placement scheme. As we show later, this LB will be sufficient to demonstrate the significant gains attainable with feedback-aided policies. Specifically, in this scheme, the source first allocates the $\beta$ portion of the pilot symbols (i.e., $\beta N_{p}$ of the $N_{p}$ pilot symbols) to a set of equally spaced subcarriers, i.e., the set $\mathcal{P}_{\mathrm{S}, 1}^{\mathrm{FB}}=\left\{0,\left\lfloor N /\left(\beta N_{p}\right)\right\rfloor, \ldots,\left(\beta N_{p}-\right.\right.$ 1) $\left.\left\lfloor N /\left(\beta N_{p}\right)\right\rfloor\right\}$, and allocates the other $(1-\beta) N_{p}$ pilots to the set of subcarriers that correspond to the $(1-\beta) N_{p}$ smallest values of $\left|\hat{H}_{\mathrm{SR}, k}\right|^{2}$, which is denoted by the set $\mathcal{P}_{\mathrm{S}, 2}^{\mathrm{FB}}$. Similarly, at the relay, $\beta N_{p}$ pilots are also allocated to the set of equalspaced subcarriers, i.e., $\mathcal{P}_{\mathrm{R}, 1}^{\mathrm{FB}}=\mathcal{P}_{\mathrm{S}, 1}^{\mathrm{FB}}$, and the other $(1-\beta) N_{p}$ pilots are allocated to the set of subcarriers with the $(1-\beta) N_{p}$ smallest values of $\left|\hat{H}_{\mathrm{RD}, k}\right|^{2}$, which is denoted by the set $\mathcal{P}_{\mathrm{R}, 2}^{\mathrm{FB}}$. Note that the effective SNR is affected by two factors: the channel estimation errors and the transmission quality of data subcarriers. Placing pilots on equal-spaced subcarriers yields smaller channel estimation errors, whereas placing them on subcarriers with small channel gains allows subcarriers with large channel gains to be left for data transmission. As a result, large $\beta$ leads to smaller estimation error (since more percentage of pilots are equal-spaced) but small $\beta$ will allow us to leave more good subcarriers for data transmission. If only the $\beta N_{p}$ equally-spaced pilot symbols are taken to perform channel estimation, the estimation errors on the $k$ th subcarrier of the SR and $\mathrm{RD}$ channels will be equal to $\sum_{\ell=0}^{L_{1}-1} 1 /\left(1 / \sigma_{\mathrm{SR}, \ell}^{2}+\beta N_{p} \gamma_{p}\right)$ and $\sum_{\ell=0}^{L_{2}-1} 1 /\left(1 / \sigma_{\mathrm{RD}, \ell}^{2}+\beta N_{p} \gamma_{p}\right)$, respectively. These values can serve as upper bounds to the actual estimation errors $\epsilon_{\mathrm{SR}, k}$ and $\epsilon_{\mathrm{RD}, k}$.

Using the suboptimal scheme mentioned previously, we can LB the minimum effective SNR of the optimal feedback-aided scheme as

$$
\xi_{(\min )}^{\mathrm{FB}} \geq \max _{\varphi} \min _{k \in \mathcal{D}_{S}^{\mathrm{FB}}} \gamma_{d} \cdot \frac{1}{\left(\frac{\varepsilon_{1}(\beta)}{\left|\hat{H}_{\mathrm{SR}, k}\right|^{2}}\right)+\left(\frac{\varepsilon_{2}(\beta)}{\left|\hat{H}_{\mathrm{RD}, \varphi(k)}\right|^{2}}\right)}
$$

where $\varepsilon_{1}(\beta)=1+\gamma_{d} \cdot \sum_{\ell=0}^{L_{1}-1} 1 /\left(1 / \sigma_{\mathrm{SR}, \ell}^{2}+\beta N_{p} \gamma_{p}\right), \varepsilon_{2}(\beta)=$ $1+\gamma_{d} \cdot \sum_{\ell=0}^{L_{2}-1} 1 /\left(1 / \sigma_{\mathrm{RD}, \ell}^{2}+\beta N_{p} \gamma_{p}\right), \quad$ and $\quad \mathcal{D}_{S}^{\mathrm{FB}}=\mathcal{N} \backslash$ $\left(\mathcal{P}_{\mathrm{S}, 1}^{\mathrm{FB}} \cup \mathcal{P}_{\mathrm{S}, 2}^{\mathrm{FB}}\right)$. Note that the set of data subcarriers $\mathcal{D}_{S}^{\mathrm{FB}}$ is a function of the estimated channel gains in feedback-aided schemes. By (15) and (16), the feedback gain defined in (12) can be lower bounded as

$$
\mathcal{G}^{\mathrm{FB}} \geq \frac{\mathrm{E}\left[\max _{\varphi} \min _{k \in \mathcal{D}_{S}^{\mathrm{FB}}} \frac{1}{\left(\frac{\varepsilon_{1}(\beta)}{\left|\hat{H}_{\mathrm{SR}, k}\right|^{2}}\right)+\left(\frac{\varepsilon_{2}(\beta)}{\left|\hat{H_{\mathrm{RD}}, \varphi(k)}\right|^{2}}\right)}\right]}{\mathrm{E}\left[\max _{\varphi} \min _{k \in \mathcal{D}^{\mathrm{EQ}}} \frac{1}{\left(\frac{\varepsilon_{1}}{\left|\hat{H}_{\mathrm{SR}, k}\right|^{2}}\right)+\left(\frac{\varepsilon_{2}}{\left|\hat{H}_{\mathrm{RD}, \varphi(k)}\right|^{2}}\right)}\right]} .
$$

To facilitate our understanding of the problem, we first examine the feedback gain LB for the case without SP in Section III-A and later extend these results to the case with SP in Section III-B, where the optimal SP is also derived.

\section{A. The Case Without Subcarrier Pairing}

In the case without SP, we have $\mathcal{D}_{S}=\mathcal{D}_{R}, \mathcal{P}_{S}=\mathcal{P}_{R}$, and $\varphi$ an identity function, where $\varphi(k)=k$ for all $k \in \mathcal{D}_{S}$. By defining

$$
G(\beta)_{k} \triangleq \frac{1}{\left(\frac{\varepsilon_{1}(\beta)}{\left|\hat{H}_{\mathrm{SR}, k}\right|^{2}}\right)+\left(\frac{\varepsilon_{2}(\beta)}{\left|\hat{H}_{\mathrm{RD}, k}\right|^{2}}\right)}
$$

and $G_{k} \triangleq G(1)_{k}$, the feedback gain LB in (17) can be expressed as

$$
\begin{aligned}
& \mathcal{G}^{\mathrm{FB}} \geq \frac{\mathrm{E}\left[\min _{k \in \mathcal{D}_{S}^{\mathrm{FB}}} G(\beta)_{k}\right]}{\mathrm{E}\left[\min _{k \in \mathcal{D}^{\mathrm{EQ}}} G_{k}\right]}
\end{aligned}
$$

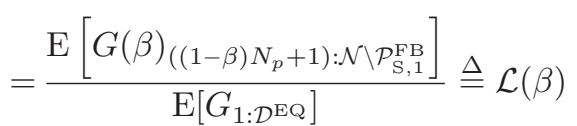

where $G_{r: \mathcal{D}}$ denotes the $r$ th-order statistic ( $r$ th smallest value) among the set $\left\{G_{k}, \forall k \in \mathcal{D}\right\}$.

Notice that $\mathrm{E}\left[G(\beta)_{\left.\left((1-\beta) N_{p}+1\right): \mathcal{N} \backslash \mathcal{P}_{\mathrm{S}, 1}^{\mathrm{FB}}\right]}\right.$ and $\mathrm{E}\left[G_{1: \mathcal{D}^{\mathrm{EQ}}}\right]$ in $\mathcal{L}(\beta)$ are difficult to evaluate in closed form due to the correlation between subcarriers. Hence, we consider the coherence block model to approximate the channel correlation and, in this way, obtain an analytic understanding of its impact on system performance. In this model, the channel coefficients are assumed to remain constant over a block containing $B$ adjacent subcarriers and are assumed to vary independently from block to block. By doing so, the LB in (19) can be approximated as

$$
\mathcal{L}(\beta) \simeq \frac{\left.\mathrm{E}\left[\tilde{G}(\beta)^{\lceil} \frac{(1-\beta) N_{p}+1}{B}\right\rceil:\left\lceil\frac{N-\beta N_{p}}{B}\right\rceil\right]}{\mathrm{E}\left[\tilde{G}(1)_{\left.1:\left\lceil\frac{N_{d}}{B}\right\rceil\right]}\right]}
$$

where $\left\{\tilde{G}(\beta)_{k}, \forall k\right\}$ is a sequence of independent identically distributed (i.i.d.) random variables with distribution equal to the marginal distribution of $G(\beta)_{k}$, and $\tilde{G}(\beta)_{r: n}$ is the $r$ thordered statistic (i.e., the $r$ th smallest value) among $n$ i.i.d. random variables $\tilde{G}(\beta)_{1}, \ldots, \tilde{G}(\beta)_{n}$. In practice, a reasonable block size will be equal to

$$
B=\frac{\text { Coherence bandwidth }}{\text { Subcarrier spacing }}=\frac{1 /\left(5 T_{\mathrm{RMS}}\right)}{1 /\left(N T_{s}\right)}=\frac{N}{c L_{h}}
$$

where $T_{\mathrm{RMS}}$ is the root mean square (RMS) delay spread, $T_{s}$ is the sampling period, $L_{h}=L_{1}+L_{2}-1$ is the overall SRD channel length, and $c$ is the constant given by $c=5$. $T_{\mathrm{RMS}} /\left(T_{s} L_{h}\right)$. The coherence bandwidth is chosen as the frequency separation that results in correlation equal to 0.5 [39].

To further evaluate the $\operatorname{LB} \mathcal{L}(\beta)$, it is necessary to derive the probability density function (pdf) of $\tilde{G}(\beta)_{k}$ (or, equivalently, the marginal distribution of $G(\beta)_{k}$ ). Since $\hat{H}_{\mathrm{SR}, k}$ and $\hat{H}_{\mathrm{RD}, k}$ are linear combinations of complex Gaussian random variables, the estimated channel gains $\left|\hat{H}_{\mathrm{SR}, k}\right|^{2}$ and $\left|\hat{H}_{\mathrm{RD}, k}\right|^{2}$ will be exponentially distributed with mean approaching $\sigma_{\mathrm{SR}}^{2}$ and $\sigma_{\mathrm{RD}}^{2}$ at high $\mathrm{SNR}$, respectively. Thus, the pdfs 
for both $H_{1} \triangleq\left|\hat{H}_{\mathrm{SR}, k}\right|^{2} / \varepsilon_{1}(\beta)$ and $H_{2} \triangleq\left|\hat{H}_{\mathrm{RD}, k}\right|^{2} / \varepsilon_{2}(\beta)$ are given by $f_{H_{1}}(h)=\tilde{\varepsilon}_{1}(\beta) e^{-\tilde{\varepsilon}_{1}(\beta) h} u(h)$ and $f_{H_{2}}(h)=$ $\tilde{\varepsilon}_{2}(\beta) e^{-\tilde{\varepsilon}_{2}(\beta) h} u(h)$, respectively, where $\tilde{\varepsilon}_{1}(\beta)=\varepsilon_{1}(\beta) / \sigma_{\mathrm{SR}}^{2}$, $\tilde{\varepsilon}_{2}(\beta)=\varepsilon_{2}(\beta) / \sigma_{\mathrm{RD}}^{2}$, and $u(h)$ is the unit-step function. The pdf of $G(\beta)_{k}$, which is defined in (18), can then be computed as

$$
\begin{aligned}
f_{G(\beta)}(g)=\tilde{\varepsilon}_{1}(\beta) & \cdot \tilde{\varepsilon}_{2}(\beta) \cdot g \int_{0}^{1} \frac{1}{t^{2}(1-t)^{2}} \\
& \times e^{-g\left(\frac{-t\left(\tilde{\varepsilon}_{1}(\beta)-\tilde{\varepsilon}_{2}(\beta)\right)+\tilde{\varepsilon}_{1}(\beta)}{t(1-t)}\right)} d t \cdot u(g)
\end{aligned}
$$

for all $k$. To obtain a closed-form expression for the integral in (21), we replace the quadratic term $t(1-t)$ with the first-order approximation $a \cdot t$ for $0 \leq t \leq 1 / 2$. This can be viewed as the first-order Taylor approximation when $a=1$. However, the value of $a$ in general can be chosen to minimize the approximation error, i.e., $\int_{0}^{1 / 2}|t(1-t)-a t|^{2} d t$, which is given by $a=0.625$ in this case. Similarly, the term $t(1-t)$ can be approximated with $0.625 \cdot(1-t)$ for $1 / 2<t \leq 1$. By doing so, the pdf in (21) can be approximated as

$$
\begin{aligned}
f_{G(\beta)}(g) \simeq & \frac{1}{(0.625)^{2}} \cdot \tilde{\varepsilon}_{1}(\beta) \cdot \tilde{\varepsilon}_{2}(\beta) \cdot g \cdot u(g) \\
& \times\left(\int_{0}^{\frac{1}{2}} \frac{1}{t^{2}} e^{-\frac{1}{0.625} \cdot g\left(\frac{-t\left(\tilde{\varepsilon}_{1}(\beta)-\tilde{\varepsilon}_{2}(\beta)\right)+\tilde{\varepsilon}_{1}(\beta)}{t}\right)} d t\right. \\
& \left.\quad+\int_{\frac{1}{2}}^{1} \frac{1}{(1-t)^{2}} e^{-\frac{1}{0.625} \cdot g\left(\frac{-t\left(\tilde{\varepsilon}_{1}(\beta)-\tilde{\varepsilon}_{2}(\beta)\right)+\tilde{\varepsilon}_{1}(\beta)}{(1-t)}\right)} d t\right) \\
= & \frac{1}{0.625} \cdot\left(\tilde{\varepsilon}_{1}(\beta)+\tilde{\varepsilon}_{2}(\beta)\right) \cdot e^{-\frac{1}{0.625} \cdot g \cdot\left(\tilde{\varepsilon}_{1}(\beta)+\tilde{\varepsilon}_{2}(\beta)\right)} u(g) .
\end{aligned}
$$

The expectation of $\tilde{G}(\beta)_{r: n}$ can then be computed as (cf. Appendix B)

$$
\begin{aligned}
\mathrm{E}\left[\tilde{G}(\beta)_{r: n}\right] \simeq & \frac{0.625}{\tilde{\varepsilon}_{1}(\beta)+\tilde{\varepsilon}_{2}(\beta)} \cdot \frac{n !}{(r-1) !(n-r) !} \\
& \times \sum_{i=0}^{r-1} \frac{(r-1) !}{i !(r-1-i) !} \cdot \frac{(-1)^{i}}{(i+n-r+1)^{2}} .
\end{aligned}
$$

The LB of the feedback gain given in (20) can then be approximated as

$$
\begin{aligned}
\mathcal{L}(\beta) \simeq & \frac{\mathrm{E}\left[\tilde{G}(\beta)_{\tilde{N}_{p}+1: \tilde{N}}\right]}{\mathrm{E}\left[\tilde{G}(1)_{1: \tilde{N}_{d}}\right]} \\
\simeq & \frac{\tilde{\varepsilon}_{1}(1)+\tilde{\varepsilon}_{2}(1)}{\tilde{\varepsilon}_{1}(\beta)+\tilde{\varepsilon}_{2}(\beta)} \cdot \frac{\left\lceil\frac{N_{d}}{B}\right] \tilde{N} !}{\tilde{N}_{p} !\left(\tilde{N}-\tilde{N}_{p}-1\right) !} \\
& \times \sum_{i=0}^{\tilde{N}_{p}} \frac{\tilde{N}_{p} !}{i !\left(\tilde{N}_{p}-i\right) !} \cdot \frac{(-1)^{i}}{\left(i+\tilde{N}-\tilde{N}_{p}\right)^{2}}
\end{aligned}
$$

where $\quad \tilde{N}_{d}=\left\lceil N_{d} / B\right\rceil, \quad \tilde{N}=\left\lceil\left(N-\beta N_{p}\right) / B\right\rceil, \quad$ and $\quad \tilde{N}_{p}=$ $\left\lceil\left((1-\beta) N_{p}+1\right) / B\right\rceil-1$. By assuming that $\tilde{N} \gg \tilde{N}_{p}$, the LB can further be approximated as

$$
\begin{aligned}
\mathcal{A}(\beta)=\frac{\tilde{\varepsilon}_{1}(1)+\tilde{\varepsilon}_{2}(1)}{\tilde{\varepsilon}_{1}(\beta)+\tilde{\varepsilon}_{2}(\beta)} & \\
& \cdot \frac{\left\lceil\frac{N_{d}}{B}\right\rceil}{\tilde{N}-\tilde{N}_{p}}\left(\tilde{N}_{p}+1\right)\left(1+\frac{1}{\tilde{N}-\tilde{N}_{p}}\right)^{\tilde{N}_{p}} .
\end{aligned}
$$

The details are given in Appendix C.

For special case where $L_{1}=L_{2}=L$ and $\sigma_{\mathrm{SR}, \ell}^{2}=\sigma_{\mathrm{SR}}^{2} / L$, $\sigma_{\mathrm{RD}, \ell}^{2}=\sigma_{\mathrm{RD}}^{2} / L$ for $\ell=0, \ldots, L-1$, we have $\varepsilon_{1}(\beta) \simeq(1+$ $\left.L \gamma_{d} /\left(\beta N_{p} \gamma_{p}\right)\right) / \sigma_{\mathrm{SR}}^{2}$ and $\varepsilon_{2}(\beta) \simeq\left(1+L \gamma_{d} /\left(\beta N_{p} \gamma_{p}\right)\right) / \sigma_{\mathrm{RD}}^{2}$ at high SNR, and thus, the approximate LB $\mathcal{A}(\beta)$ in (25) can be reduced to

$$
\mathcal{A}(\beta)=\frac{\tilde{N}_{d}}{\tilde{N}-\tilde{N}_{p}}\left(\tilde{N}_{p}+1\right)\left(1+\frac{1}{\tilde{N}-\tilde{N}_{p}}\right)^{\tilde{N}_{p}} \cdot \frac{1+\frac{L \gamma_{d}}{N_{p} \gamma_{p}}}{1+\frac{L \gamma_{d}}{\beta N_{p} \gamma_{p}}} .
$$

From (26), we can see that the feedback gain increases with the number of subcarriers, i.e., $N$, with the asymptotic increase rate given by

$$
\lim _{N \rightarrow \infty} \frac{\mathcal{A}(\beta)}{N}=c \cdot \ell \cdot \alpha(1-\beta) e^{\frac{\alpha(1-\beta)}{1-\alpha}} \cdot \frac{1+\frac{\ell \gamma_{d}}{\alpha \gamma_{p}}}{1+\frac{\ell \gamma_{d}}{\alpha \beta \gamma_{p}}}
$$

where $\alpha=N_{p} / N, \ell=L / N$, and $c=5 \cdot T_{\mathrm{RMS}} /\left(T_{s} L\right)$. For a fixed number of subcarriers, we can observe that the approximated feedback gain $\mathcal{A}(\beta)$ increases with the number of pilots $N_{p}$ (or, equivalently, the parameter $\alpha$ ). Notice that the LBs given above are derived for a given value of $\beta$ but can be tightened by maximizing over the choice of $\beta$.

\section{B. The Case With Subcarrier Pairing}

In this section, we extend the analysis to systems with SP. This problem is considerably more challenging since SP and pilot placement operations are closely coupled and, thus, must be solved jointly. However, using the proposition below, we can first derive explicitly the optimal SP for any given pilot placement.

Proposition 1: Let $\varphi: \mathcal{A}=\left\{a_{0}, \ldots, a_{n-1}\right\} \rightarrow \mathcal{B}=\left\{b_{0}\right.$, $\left.\ldots, b_{n-1}\right\}$ be a one-to-one mapping with $a_{0} \leq \cdots \leq a_{n-1}$ and $b_{0} \leq \cdots \leq b_{n-1}$, and let $f(x, y)$ be a function that is monotonically nondecreasing with respect to each parameter when the other is fixed, i.e., $f\left(x_{1}, y\right) \leq f\left(x_{2}, y\right)$ for $x_{1} \leq x_{2}$ and $f\left(x, y_{1}\right) \leq f\left(x, y_{2}\right)$ for $y_{1} \leq y_{2}$. The value of $\min _{k} f\left(a_{k}, \varphi\left(a_{k}\right)\right)$ is maximized when $\varphi$ is chosen to be the inverse mapping between $\mathcal{A}$ and $\mathcal{B}$, i.e., $\varphi\left(a_{k}\right)=b_{n-1-k}$, for $k=0, \ldots, n-1$.

The proof is given in Appendix D. Note that, since the effective SNR expression in (13) can be viewed as a function of $\left|\hat{H}_{\mathrm{SR}, k}\right|^{2} /\left(1+\gamma_{d} \epsilon_{\mathrm{SR}, k}\right)$ and $\left|\hat{H}_{\mathrm{RD}, m}\right|^{2} /\left(1+\gamma_{d} \epsilon_{\mathrm{RD}, m}\right)$ that satisfies the conditions in Proposition 1, we can infer from the proposition that the optimal SP, for a given choice of $\mathcal{D}_{S}$ and $\mathcal{D}_{R}$, should be the inverse mapping between subcarriers at the source and the relay according to their effective SNRs. That is, 
the data received on the subcarrier with the smallest effective SNR on the SR link should be forwarded on the subcarrier with the largest effective SNR on the RD link, and the data received on the second worst subcarrier on the SR link should be forwarded on the second best subcarrier on the RD link, and so on. Note that the inverse mapping is opposite to the ordered mapping scheme often considered in the literature, e.g., in [8], [10], and [11]. This is due to the different objectives that were adopted. In fact, the inverse mapping is shown to maximize the minimum effective SNR, whereas the ordered mapping was shown to maximize the sum rate [8], [11] and the sum SNR [10].

By (15) and the optimal SP scheme derived from Proposition 1, the minimum effective SNR achieved with the equal-spaced pilot placement scheme can be expressed as

$$
\xi_{(\min )}^{\mathrm{EQ}}=\min _{i \in\left\{1, \ldots, N_{d}\right\}} \gamma_{d} \cdot \frac{1}{\frac{\varepsilon_{1}}{\left|\hat{H}_{\mathrm{SR}}\right|_{i: \mathcal{D}}^{2} \mathrm{EQ}}+\frac{\varepsilon_{2}}{\left|\hat{H}_{\mathrm{RD}}\right|_{\left(N_{d}+1-i\right): \mathcal{D}}^{2} \mathrm{EQ}}}
$$

where $\left|\hat{H}_{\mathrm{SR}}\right|_{r: \mathcal{D}^{\mathrm{EQ}}}^{2}$ is the $r$ th order statistic among the set $\left\{\left|\hat{H}_{\mathrm{SR}, k}\right|^{2}, \forall k \in \mathcal{D}^{\mathrm{EQ}}\right\}$, and $\left|\hat{H}_{\mathrm{RD}}\right|_{r: \mathcal{D}^{\mathrm{EQ}}}^{2}$ is the $r$ th order statistic among $\left\{\left|\hat{H}_{\mathrm{RD}, k}\right|^{2}, \forall k \in \mathcal{D}^{\mathrm{EQ}}\right\}$. Notice that the SR channel with the $i$ th smallest channel gain is matched with the RD channel with the $i$ th largest channel gain. Similarly, by (16) and the optimal SP scheme, the minimum effective SNR of the feedback-aided scheme can be lower bounded as

$$
\begin{aligned}
& \xi_{(\min )}^{\mathrm{FB}} \geq \min _{i \in\left\{1, \ldots, N_{d}\right\}} \gamma_{d} \\
& \times \frac{1}{\left(\frac{\varepsilon_{1}(\beta)}{\left|\hat{H}_{\mathrm{SR}}\right|_{\left((1-\beta) N_{p}+i\right): \mathcal{N} \backslash \mathcal{P}_{\mathrm{S}, 1}^{\mathrm{FB}}}^{2}}\right)+\left(\frac{\varepsilon_{2}(\beta)}{\left|\hat{H}_{\mathrm{RD}}\right|_{\left(N-\beta N_{p}+1-i\right): \mathcal{N} \backslash \mathcal{P}_{\mathrm{R}, 1}^{\mathrm{FB}}}^{2}}\right)}
\end{aligned}
$$

Hence, by (28) and (29), the feedback gain for the case with SP can then be lower bounded as (30), shown at the bottom of the page.

Note that the statistics of the effective SNRs, as given in the right-hand sides of (28) and (29), are difficult to obtain in closed form. However, a deterministic approximation of the expected values can help us gain intuition on the achievable feedback gain. This approximation is obtained by assigning deterministic and representative values to the channel gains, as to be shown in the following. The coherence block approximation is also employed to take into consideration the effect of channel correlation. The accuracy of the deterministic approximation will be demonstrated through computer simulations in Section V.
Specifically, let us consider the case where $L_{1}=L_{2}=L$ and $\varepsilon_{1}=\varepsilon_{2}=\varepsilon$. By the coherence block approximation, we can first approximate (28) as

$$
\xi_{(\min )}^{\mathrm{EQ}} \simeq \min _{i \in\left\{1, \ldots, \tilde{N}_{d}\right\}} \frac{\gamma_{d}}{\varepsilon} \cdot \frac{1}{\left(\frac{1}{Q_{\mathrm{SR}, i: \tilde{N}_{d}}}\right)+\left(\frac{1}{Q_{\mathrm{RD},\left(\tilde{N}_{d}+1-i\right): \tilde{N}_{d}}}\right)}
$$

where $\tilde{N}_{d}=\left\lceil N_{d} / B\right\rceil$, and $B$ is the coherence block. Here, $Q_{\mathrm{SR}, 0}, \ldots, Q_{\mathrm{SR}, \tilde{N}_{d}-1}$ are i.i.d. random variables with the distribution of $Q_{\mathrm{SR}, k}$ equal to the marginal distribution of $\left|\hat{H}_{\mathrm{SR}, k}\right|^{2}$, and $Q_{\mathrm{SR}, r: n}$ is the $r$ th-ordered statistic among the $n$ i.i.d. random variables $Q_{\mathrm{SR}, 1}, \ldots, Q_{\mathrm{SR}, n}$. The random variables $Q_{\mathrm{RD}, 0}, \ldots, Q_{\mathrm{RD}, \tilde{N}_{d}-1}$ are defined similarly. To analyze the achievable feedback gain, we approximate the ordered sequence of channel gains $Q_{\mathrm{SR}, 1: \tilde{N}_{d}}, \ldots, Q_{\mathrm{SR}, \tilde{N}_{d}: \tilde{N}_{d}}$ by the deterministic sample values $v_{1}, \ldots, v_{\tilde{N}_{d}}$, where $v_{i}$ is chosen such that $F_{Q_{\mathrm{SR}}}\left(v_{i}\right)=(2 i-1) /\left(2 \tilde{N}_{d}\right)$ for $i=1, \ldots, \tilde{N}_{d}$. Notice that if we divide $Q_{\mathrm{SR}, k}$ 's region of support into $\tilde{N}_{d}$ intervals with equal probability, the points $v_{1}, \ldots, v_{\tilde{N}_{d}}$ can be viewed as representative points in each interval. Since the pdf of $Q_{\mathrm{SR}, k}$ is given by $\left(1 / \sigma_{\mathrm{SR}}^{2}\right) \cdot e^{-q / \sigma_{\mathrm{SR}}^{2}} u(q)$, the representative values are given by

$$
v_{i}=-\sigma_{\mathrm{SR}}^{2} \cdot \ln \left(1-\frac{2 i-1}{2 \tilde{N}_{d}}\right), \text { for } i=1, \ldots, \tilde{N}_{d} .
$$

Similarly, we can also approximate the ordered sequence $Q_{\mathrm{RD}, 1: \tilde{N}_{d}}, \ldots, Q_{\mathrm{RD}, \tilde{N}_{d}: \tilde{N}_{d}}$ by $v_{1}^{\prime}, \ldots, v_{\tilde{N}_{d}}^{\prime}$, where $v_{i}^{\prime}=-\sigma_{\mathrm{RD}}^{2}$. $\ln \left(1-(2 i-1) /\left(2 \tilde{N}_{d}\right)\right)$. Therefore, the minimum effective SNR of the equal-spaced scheme can be approximated by

$$
\begin{aligned}
& \xi_{(\min )}^{\mathrm{EQ}} \simeq \min _{i \in\left\{1, \ldots, \tilde{N}_{d}\right\}} \frac{\gamma_{d}}{\varepsilon} \cdot \frac{1}{\left(\frac{1}{v_{i}}\right)+\left(\frac{1}{v_{\tilde{N}_{d}^{+1-i}}^{\prime}}\right)} \\
& =\min _{i \in\left\{1, \ldots, \tilde{N}_{d}\right\}} \frac{\gamma_{d}}{\varepsilon} \cdot \frac{1}{-\sigma_{\mathrm{SR}}^{2} \cdot \ln \left(1-\frac{2 i-1}{2 \tilde{N}_{d}}\right)}+\frac{1}{-\sigma_{\mathrm{RD}}^{2} \cdot \ln \left(\frac{2 i-1}{2 \tilde{N}_{d}}\right)} \\
& =\min _{i \in\left\{1, \ldots, \tilde{N}_{d}\right\}} \frac{\gamma_{d}}{\varepsilon} \cdot \frac{\sigma_{\mathrm{SR}}^{2} \sigma_{\mathrm{RD}}^{2} \cdot \ln \left(1-\frac{2 i-1}{2 \tilde{N}_{d}}\right) \cdot \ln \left(\frac{2 i-1}{2 \tilde{N}_{d}}\right)}{-\sigma_{\mathrm{SR}}^{2} \cdot \ln \left(1-\frac{2 i-1}{2 \tilde{N}_{d}}\right)-\sigma_{\mathrm{RD}}^{2} \cdot \ln \left(\frac{2 i-1}{2 \tilde{N}_{d}}\right)} \\
& =\left\{\begin{array}{l}
\frac{\gamma_{d}}{\varepsilon} \cdot \frac{1}{-\sigma_{\mathrm{SR}}^{2} \cdot \ln \left(1-\frac{1}{2 \tilde{N}_{d}}\right)}+\frac{1}{-\sigma_{\mathrm{RD}}^{2} \cdot \ln \left(\frac{1}{2 \tilde{N}_{d}}\right)}, \text { for } \sigma_{\mathrm{SR}}^{2} \leq \sigma_{\mathrm{RD}}^{2} \\
\frac{\gamma_{d}}{\varepsilon} \cdot \frac{1}{-\frac{1}{-\sigma_{\mathrm{SR}}^{2} \cdot \ln \left(\frac{1}{2 \tilde{N}_{d}}\right)}+\frac{1}{-\sigma_{\mathrm{RD}}^{2} \cdot \ln \left(1-\frac{1}{2 \tilde{N}_{d}}\right)}}, \text { for } \sigma_{\mathrm{SR}}^{2}>\sigma_{\mathrm{RD}}^{2}
\end{array}\right.
\end{aligned}
$$

where proof of the last equality is given in Appendix E.

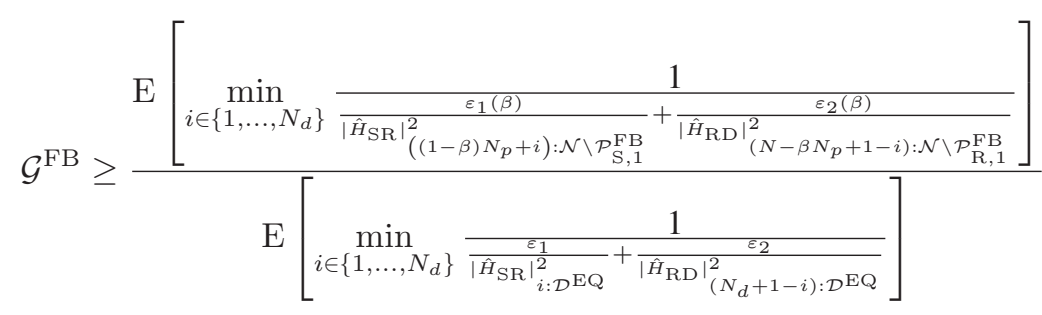


The same approach can also be used to approximate the minimum effective SNR of the feedback-aided schemes given in (29). Specifically, by applying the coherent block approximation, the LB of $\xi_{(\min )}^{\mathrm{FB}}$ in (29) can first be computed as

$\xi_{(\min )}^{\mathrm{FB}} \geq \min _{i \in\left\{1, \ldots, \tilde{N}-\tilde{N}_{p}\right\}} \frac{\gamma_{d}}{\varepsilon(\beta)} \cdot \frac{1}{\left(\frac{1}{Q_{\mathrm{SR},\left(\tilde{N}_{p}+i\right): \tilde{N}}}\right)+\left(\frac{1}{Q_{\mathrm{RD},(\tilde{N}+1-i): \tilde{N}}}\right)}$

where $\varepsilon_{1}(\beta)=\varepsilon_{2}(\beta)=\varepsilon(\beta), \tilde{N}_{p}=\left\lceil\left((1-\beta) N_{p}+1\right) / B\right\rceil-$ 1 , and $\tilde{N}=\left\lceil\left(N-\beta N_{p}\right) / B\right\rceil$. Then, by again considering the deterministic approximation, the ordered sequence $Q_{\mathrm{SR}, 1: \tilde{N}}, \ldots, Q_{\mathrm{SR}, \tilde{N}: \tilde{N}}$ can be approximated by the deterministic sample values $w_{1}, \ldots, w_{\tilde{N}}$, where $w_{i}=-\sigma_{\mathrm{SR}}^{2} \cdot \ln (1-$ $(2 i-1) /(2 \tilde{N}))$ for $i=1, \ldots, \tilde{N}$, and similarly for the ordered sequence $Q_{\mathrm{RD}, 1: \tilde{N}}, \ldots, Q_{\mathrm{RD}, \tilde{N}: \tilde{N}}$ given by $w_{1}^{\prime}, \ldots, w_{\tilde{N}}^{\prime}$, where $w_{i}^{\prime}=-\sigma_{\mathrm{RD}}^{2} \cdot \ln (1-(2 i-1) /(2 \tilde{N}))$. In this case, we have

$$
\begin{aligned}
& \xi_{(\min )}^{\mathrm{FB}} \geq \min _{i \in\left\{1, \ldots, \tilde{N}-\tilde{N}_{p}\right\}} \frac{\gamma_{d}}{\varepsilon(\beta)} \cdot \frac{1}{\left(\frac{1}{w_{\tilde{N}_{p}+i}}\right)+\left(\frac{1}{w_{\tilde{N}+1-i}^{\prime}}\right)} \\
& =\left\{\begin{array}{l}
\frac{\gamma_{d}}{\varepsilon(\beta)} \cdot \frac{1}{-\sigma_{\mathrm{SR}}^{2} \cdot \ln \left(1-\frac{2 \tilde{N}_{p}+1}{2 \tilde{N}}\right)}+\frac{1}{-\sigma_{\mathrm{RD}}^{2} \cdot \ln \left(\frac{1}{2 \tilde{N}}\right)}, \text { for } \sigma_{\mathrm{SR}}^{2} \leq \sigma_{\mathrm{RD}}^{2} \\
\frac{\gamma_{d}}{\varepsilon(\beta)} \cdot \frac{1}{-\sigma_{\mathrm{SR}}^{2} \cdot \ln \left(\frac{1}{2 \tilde{N}}\right)}+\frac{1}{-\sigma_{\mathrm{RD}}^{2} \cdot \ln \left(1-\frac{2 \tilde{N}_{p}+1}{2 \tilde{N}}\right)}, \text { for } \sigma_{\mathrm{SR}}^{2}>\sigma_{\mathrm{RD}}^{2}
\end{array}\right.
\end{aligned}
$$

which also follows from Appendix E. For $\sigma_{\mathrm{SR}}^{2} \leq \sigma_{\mathrm{RD}}^{2}$, it follows from (31) and (32) that the feedback gain with SP can be approximately lower bounded by

$$
\begin{aligned}
& \mathcal{G}^{\mathrm{FB}} \geq \frac{\varepsilon}{\varepsilon(\beta)} \cdot \frac{\frac{1}{-\sigma_{\mathrm{SR}}^{2} \cdot \ln \left(1-\frac{1}{2 \tilde{N}_{d}}\right)}+\frac{1}{-\sigma_{\mathrm{RD}}^{2} \cdot \ln \left(\frac{1}{2 \tilde{N}_{d}}\right)}}{-\sigma_{\mathrm{SR}}^{2} \cdot \ln \left(1-\frac{2 \tilde{N}_{p}+1}{2 \tilde{N}}\right)}+\frac{1}{-\sigma_{\mathrm{RD}}^{2} \cdot \ln \left(\frac{1}{2 \tilde{N}}\right)} \\
& \stackrel{(a)}{\approx} \frac{\varepsilon}{\varepsilon(\beta)} \cdot \frac{\frac{1}{-\sigma_{\mathrm{SR}}^{2} \cdot \ln \left(1-\frac{1}{2 \tilde{N}_{d}}\right)}}{\frac{1}{-\sigma_{\mathrm{SR}}^{2} \cdot \ln \left(1-\frac{2 \tilde{N}_{p}+1}{2 \tilde{N}}\right)}}=\frac{\varepsilon}{\varepsilon(\beta)} \cdot \frac{\ln \left(1-\frac{2 \tilde{N}_{p}+1}{2 \tilde{N}}\right)}{\ln \left(1-\frac{1}{2 \tilde{N}_{d}}\right)} \\
& \quad \stackrel{(b)}{\approx} \frac{\varepsilon}{\varepsilon(\beta)} \cdot \frac{\left(\frac{2 \tilde{N}_{p}+1}{2 \tilde{N}}\right)}{\left(\frac{1}{2 \tilde{N}_{d}}\right)}=\frac{\tilde{N}_{d}}{\tilde{N}} \cdot\left(2 \tilde{N}_{p}+1\right) \cdot \frac{1+\frac{L \gamma_{d}}{N_{p} \gamma_{p}}}{1+\frac{L \gamma_{d}}{\beta N_{p} \gamma_{p}}} \\
& \quad \triangleq \mathcal{A}^{\mathrm{SP}}(\beta)
\end{aligned}
$$

where (a) follows by assuming that $\tilde{N}_{d}$ and $\tilde{N}$ are sufficiently large, and $(b)$ follows by taking the first-order Taylor series expansion of $\ln (1-x)$ about $x=0$. Note that the $\mathcal{A}^{\mathrm{SP}}(\beta)$ given in (33) is the same for the case where $\sigma_{\mathrm{SR}}^{2}>\sigma_{\mathrm{RD}}^{2}$. From this approximate LB, we can see that the feedback gain increases with the number of subcarriers, i.e., $N$, with the asymptotic rate of increase given by

$$
\lim _{N \rightarrow \infty} \frac{\mathcal{A}^{\mathrm{SP}}(\beta)}{N}=c \cdot \ell \cdot 2 \alpha(1-\beta) \cdot \frac{1-\alpha}{1-\alpha \beta} \cdot \frac{1+\frac{\ell \gamma_{d}}{\alpha \gamma_{p}}}{1+\frac{\ell \gamma_{d}}{\alpha \beta \gamma_{p}}} .
$$

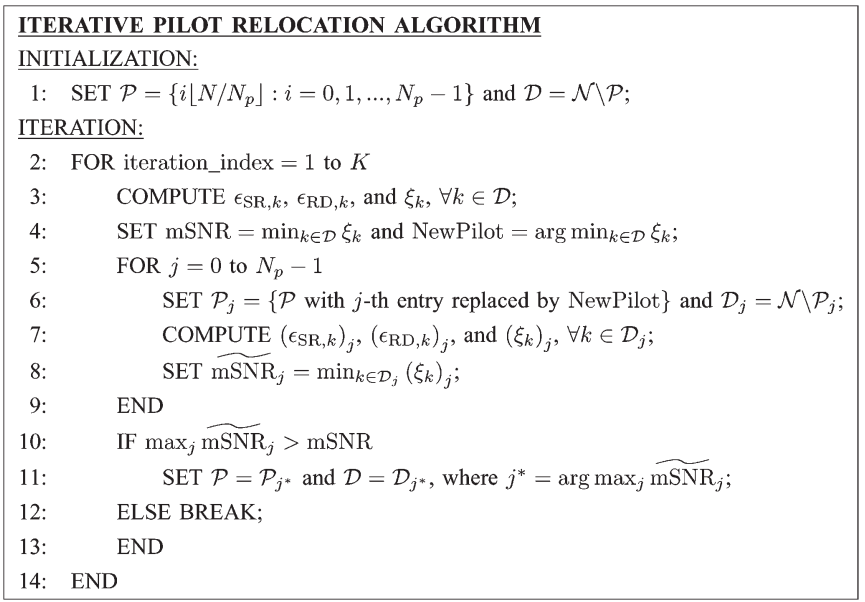

Fig. 2. Proposed IterPR algorithm for OFDM relay systems without SP.

Recall that $\alpha=N_{p} / N, \ell=L / N$, and $c=5 \cdot T_{\mathrm{RMS}} /\left(T_{s} L\right)$. By comparing (34) with (27), we can see that the approximate feedback gains increase faster with $N$ when the system employs SP. Although the feedback gain LB is analyzed using a deterministic approximation, we show in Section $\mathrm{V}$ that the results actually closely approximate the LB obtained through Monte Carlo simulations.

The analytic LBs obtained in this section provide good insights on the gain achievable with feedback-aided schemes. In particular, the closed-form approximations can be useful in evaluating the performance of systems with large $N$ since the optimal feedback-aided policy may have intractable complexity in these cases. However, the suboptimal decoupled scheme used to derive these bounds can be improved upon considerably compared to the optimal scheme (cf. Section V). To better exploit the advantages of feedback and to avoid the intractability of the optimal scheme, we propose in the following section a suboptimal but efficient pilot placement scheme for use in practical systems.

\section{Iterative Pilot Relocation (IterPR) Policy}

In this section, IterPR policies are proposed for systems without and with SP. The key idea is to iteratively exchange locations between one of the pilot subcarriers and the worst data subcarrier so that the system's minimum effective SNR can be gradually improved through the process.

Specifically, let us first describe the IterPR algorithm for systems without SP, i.e., the case where the source and relay use the same sets of pilot and data subcarriers. Here, we set $\mathcal{D}_{S}=\mathcal{D}_{R}=\mathcal{D}$ and $\mathcal{P}_{S}=\mathcal{P}_{R}=\mathcal{P}$. The pseudocode of the algorithm is given in Fig. 2. In particular, as indicated in Line 1 of the pseudocode, the IterPR algorithm first initializes the pilot set with equally spaced pilot subcarriers, which is denoted by $\mathcal{P}=\left\{i\left\lfloor N / N_{p}\right\rfloor: i=0,1, \ldots, N_{p}-1\right\}$. Given the current set of data subcarriers $\mathcal{D}=\mathcal{N} \backslash \mathcal{P}$, the estimation error variances $\epsilon_{\mathrm{SR}, k}$ and $\epsilon_{\mathrm{RD}, k}$ are then computed from (6) and (7), respectively, for all $k \in \mathcal{D}$, and utilized to evaluate the minimum effective SNR among all data subcarriers, i.e., $\min _{k \in \mathcal{D}} \xi_{k}$. In each stage of the iteration process, we in turn exchange each pilot subcarrier with the data subcarrier that currently has the minimum effective SNR and compute the minimum effective 


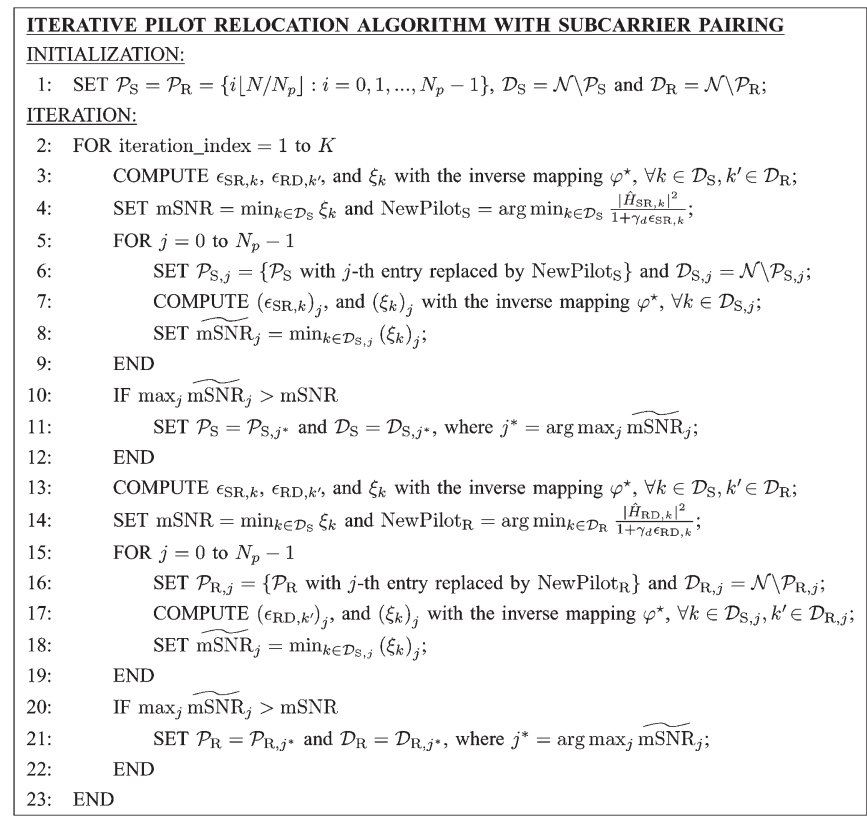

Fig. 3. Proposed IterPR algorithm for OFDM relay systems that employ SP.

SNR resulting from each possible exchange (as shown in Lines 5-9). This yields the set of values $\widetilde{\operatorname{mSNR}}_{j}$ for $j=$ $0, \ldots, N_{p}-1$. The pilot set that results in the largest minimum effective SNR, i.e., $\mathcal{P}_{j^{*}}$, is chosen and used to update the pilot set $\mathcal{P}$ if its minimum effective $\mathrm{SNR}$, i.e., $\widetilde{\mathrm{mSNR}}_{j^{*}}$, exceeds that of the original set (see Lines 10-13). The channel estimation error variances as well as the minimum effective SNR are updated accordingly. Repeating the foregoing procedure will lead to increases in the minimum effective SNR. Note that the number of iterations generally required to yield an effective solution for the IterPR algorithm is less than $N_{p}$ and can be made much smaller than $N_{p}$ if the channel is highly correlated and if the pilot placement obtained in the previous frame is used in the initialization instead of the equal-spaced scheme (cf. Section V). Hence, the complexity of $K N_{p}$ required for the IterPR algorithm is much smaller than that required for the optimal scheme, which is $\left(\begin{array}{c}N \\ N_{p}\end{array}\right)$. The IterPR policy can also be applied in systems employing other design criteria, i.e., by simply replacing the minimum effective SNR, i.e., mSNR, with criteria such as average SNR, average error probability, etc.

The proposed IterPR algorithm can be extended to systems with SP, as shown in Fig. 3. When SP is employed, the pilot locations may be different between the source and the relay. Therefore, the exchange of pilot and data subcarriers required in each iteration of the IterPR algorithm must be performed separately for the source and relay. Yet, after each exchange of pilot tones at either the source or the relay, the pairing of data subcarriers is updated according to the inverse mapping between the sets $\mathcal{D}_{S}$ and $\mathcal{D}_{R}$, as derived from Proposition 1. Specifically, in each iteration of the process, we first update the pilot locations at the source, that is, we in turn exchange each pilot subcarrier in $\mathcal{P}_{S}$ with the data subcarrier in $\mathcal{D}_{S}$ that currently has the minimum equivalent $\mathrm{SR}$ channel gain $\hat{H}_{\mathrm{SR}, k} /\left(1+\gamma_{d} \epsilon_{\mathrm{SR}, k}\right)$. Then, similar to the previous case, only the exchange that yields the largest effective channel gain and that improves the minimum effective SNR $\xi_{k}$ will be adopted (cf. Lines 3-12 in Fig. 3). The update of pilot locations at the relay, i.e., $\mathcal{P}_{R}$, is performed following the same procedure, as described in Lines 13-22 of the pseudocode. It is worthwhile to mention that for the case with SP, the optimal pilot placement scheme requires complexity of $\left(\begin{array}{c}N \\ N_{p}\end{array}\right)^{2}$, whereas the IterPR algorithm requires complexity of only $2 K N_{p}$ in the case with SP.

Remark 2: It is interesting to remark that the proposed IterPR algorithms in Figs. 2 and 3 need not be initialized with the equal-spaced pilot placement scheme (as done in Line 1 of both pseudocodes). Instead, it can be replaced with more effective placement schemes, such as the decoupled scheme (where $\beta N_{p}$ pilots are equally spaced, and $(1-\beta) N_{p}$ pilots are placed on the remaining worst subcarriers). The IterPR algorithm guarantees performance better than its initialization scheme since the pilot placement in each iteration is updated only if there is an improvement compared to that of the previous iteration. However, initializing with more advanced schemes may require higher complexity, and thus, the equal-spaced scheme is considered here.

Remark 3: The IterPR scheme given in this section is derived based on the effective SNR of the SR and RD links. However, if the direct link between the source and the destination is also considered, the effective SNR can be written as

$$
\xi_{k}=\frac{\left|\hat{H}_{\mathrm{SD}, k}\right|^{2}}{\epsilon_{\mathrm{SD}, k}+\frac{1}{\gamma_{d}}}+\frac{\gamma_{d}\left|\hat{H}_{\mathrm{SR}, k}\right|^{2} \cdot\left|\hat{H}_{\mathrm{RD}, \varphi(k)}\right|^{2}}{\left|\hat{H}_{\mathrm{SR}, k}\right|^{2}+\left|\hat{H}_{\mathrm{RD}, \varphi(k)}\right|^{2}+\zeta_{k}+\frac{1}{\gamma_{d}}}
$$

where $\hat{H}_{\mathrm{SD}, k}$ is the estimated source-to-destination (SD) channel on subcarrier $k$, and $\epsilon_{\mathrm{SD}, k}$ is the corresponding estimation error variance. The first term in (35) is the effective SNR of the direct link. The IterPR algorithm can also be applied in this case by replacing the effective SNR with the one given in (35). Following the same procedure, the IterPR algorithm can be applied to other design criteria (and even for DF systems [11], [15]-[18] as well), but the optimal SP may not be the inverse mapping in these cases. Studies on SP under different design criteria can be found in [8] and [11].

\section{Simulation Results}

In this section, we demonstrate the effectiveness of the proposed feedback-aided pilot placement scheme through computer simulations. The powers assigned to each data and pilot symbols are assumed to be the same, i.e., $\rho_{d}=\rho_{p}=$ $\rho$. We consider a time-varying multipath channel for both SR and RD links. The channel impulse responses $\mathbf{h}_{\mathrm{SR}}$ and $\mathbf{h}_{\mathrm{RD}}$ are given by the exponential decay profile [40], where $\sigma_{\mathrm{SR}, \ell_{1}}^{2}=\sigma_{\mathrm{SR}, 0}^{2} e^{-2 \ell_{1} / L_{1}}$ and $\sigma_{\mathrm{RD}, \ell_{2}}^{2}=\sigma_{\mathrm{RD}, 0}^{2} e^{-2 \ell_{2} / L_{2}}$ for $\ell_{1}=$ $0, \ldots, L_{1}-1$ and $\ell_{2}=0, \ldots, L_{2}-1$. The channel RMS delay spread is $T_{\mathrm{RMS}}=100 \mathrm{~ns}$, and the channel coherence bandwidth is $1 /\left(5 T_{\mathrm{RMS}}\right)=2 \mathrm{MHz}$ (defined such that correlation is above 0.5 within the coherence bandwidth [39]). The normalized Doppler frequency is given by $F_{d} \triangleq f_{d} N T_{s}=0.001$, where $T_{s}=50 \mathrm{~ns}$ is the sampling period, and $f_{d}$ is the channel Doppler frequency given by $312.5 \mathrm{~Hz}$ for $N=64$ systems. The system bandwidth is $1 / T_{s}=1 /(50 \mathrm{~ns})=20 \mathrm{MHz}$. The cyclic prefix is set to be $1 / 4$ OFDM symbol periods, and in the simulations of $N=64$, the cyclic prefix is 16 times the 


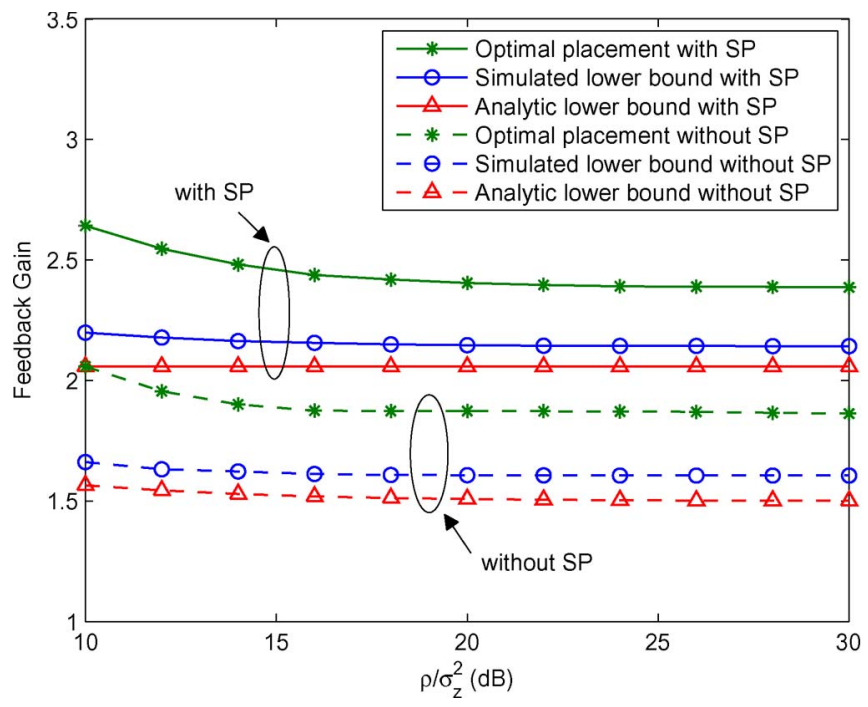

Fig. 4. Analytic LB, simulated LB, and optimal feedback gain obtained via exhaustive search for a system with $N=12, N_{p}=3$, and $L_{1}=L_{2}=3$. Solid and dashed lines represent cases with and without SP, respectively.

sampling period. The channel in each OFDM symbol period is generated by using Jakes model [41]. We assume that the channel estimates are fed back once every five OFDM symbol periods, during which the pilot locations are selected according to the proposed IterPR algorithm. For the SER simulations, we consider uncoded OFDM systems with quadrature phase-shift keying modulation. The minimum distance detector is used when examining the SER.

First, in Fig. 4, we show, for a system with $N=12, N_{p}=3$, and $L_{1}=L_{2}=3$, the approximate $\mathrm{LB} \mathcal{A}(\beta)$ given in (25), the simulated $\operatorname{LB} \mathcal{L}(\beta)$ given in (19), and the feedback gain $\mathcal{G}^{\mathrm{FB}}$ of the optimal placement (obtained through exhaustive search). Here, $\beta$ is chosen among the set of possible values $0,1 / N_{p}, 2 / N_{p}, \ldots, 1$ to maximize the LBs. We can see that the approximated LBs are indeed close to the simulated LBs, even for the case with SP (where a deterministic approximation is used). The approximate LBs can be used to demonstrate the significant gains achievable with feedback-aided schemes and, more importantly, to help evaluate the performance of feedback-aided schemes when $N$ is large. However, we can also observe from Fig. 4 that the gains achievable with the optimal scheme is substantially higher than the LB that is derived based on the decoupled scheme proposed in [29] and [30].

In Figs. 5 and 6, we examine the performance of the proposed IterPR algorithm for systems without SP. Specifically, in Fig. 5, the IterPR algorithm is compared with three other schemes: 1) the optimal pilot placement scheme (which is obtained through exhaustive search); 2) the equal-spaced pilot placement scheme; and 3) the decoupled feedback-aided pilot placement scheme [29] (which places $\beta N_{p}$ pilots on equally spaced subcarriers and $(1-\beta) N_{p}$ pilots on the remaining subcarriers with the worst effective SNRs, i.e., $G_{k}$ ). Here, the performance is measured by the average minimum effective SNR among data subcarriers, and the optimal $\beta$ is chosen for the decoupled scheme for all SNRs. Due to the complexity of the exhaustive search, we consider only a small-scale system with $N=16$ and $N_{p}=4$. From the figure, we can see that the proposed IterPR algorithm performs close to the optimal pilot placement

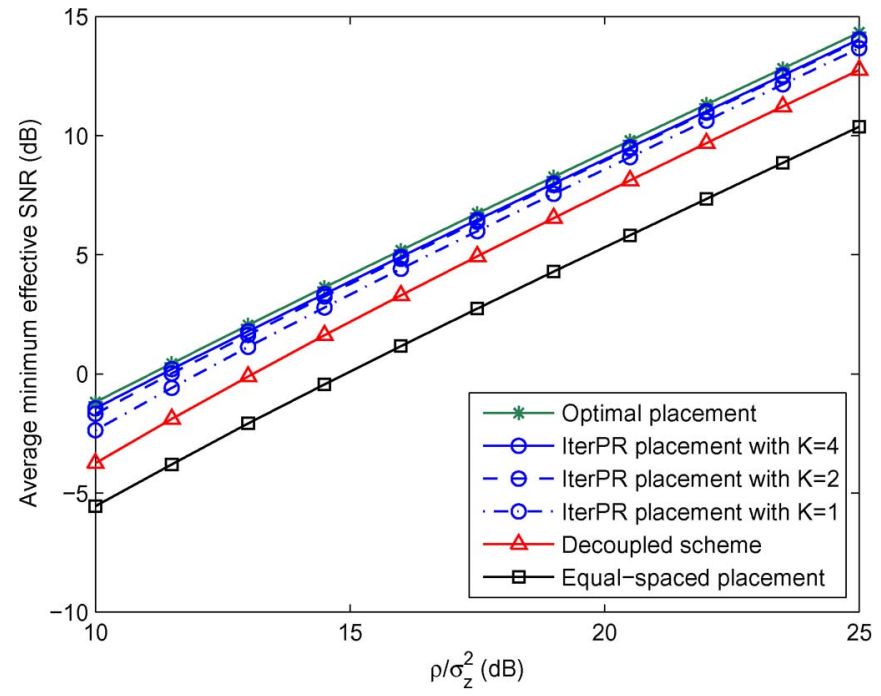

Fig. 5. Average minimum effective SNR for different pilot placement schemes with $N=16, N_{p}=4, L_{1}=3$, and $L_{2}=2$.

scheme and improves significantly over other pilot placement schemes by using only a small number of iterations. In fact, the experimental results show that the IterPR algorithm improves rapidly as $K$ increases, and the improvement in average minimum effective SNR becomes less than $0.1 \%$ after only four iterations. Recall that two effects take place in each iteration of the IterPR algorithm: 1) the improvement of the channel gain due to the removement of the worst data subcarrier, and 2) the slight decrease in channel estimation performance due to the gradually less regular placement of pilot tones. The first effect is most significant in the first few iterations since the minimum effective SNR will be dominated by the worst channel gain on the data subcarriers in these cases. However, after removing the worst few subcarriers, the improvement in the worst channel gain of data subcarriers becomes less significant and becomes comparable with the degradation in channel estimation performance. The improvement in the minimum effective SNR is thus less evident after a few iterations. However, the required number of iterations, i.e., $K$, does increase with the number of pilot subcarriers. In Fig. 6, we show the minimum effective SNR and the SER (obtained by using the estimated channels) for a large-scale system with $N=64$ and $N_{p}=8$. Specifically, in Fig. 6(a), we observe that gains of approximately 2.4 and $4.8 \mathrm{~dB}$, in terms of the average minimum effective SNR, can be achieved with the IterPR algorithm (with $K=8$ ) compared with the decoupled and equal-spaced schemes, respectively. In Fig. 6(b), we observe that, for an error rate of $10^{-3}$, the IterPR algorithm improves over the decoupled and equalspaced schemes by approximately 2 and $5.7 \mathrm{~dB}$, respectively. The proposed scheme achieves the minimum SER among all schemes at high SNR since it is derived by maximizing the minimum effective SNR, which dominates the SER at high SNR.

In Fig. 7, we show the minimum effective SNR versus the number of iterations $K$ for a system with a large number of subcarriers (i.e., $N=1024$ and $N_{p}=64$ ). We can see that generally no improvements can be obtained after $K=N_{p}$ iterations. However, when the previous pilot placement is used as the initialization instead of the equal-spaced scheme, the number of iterations needed can be reduced substantially. This 


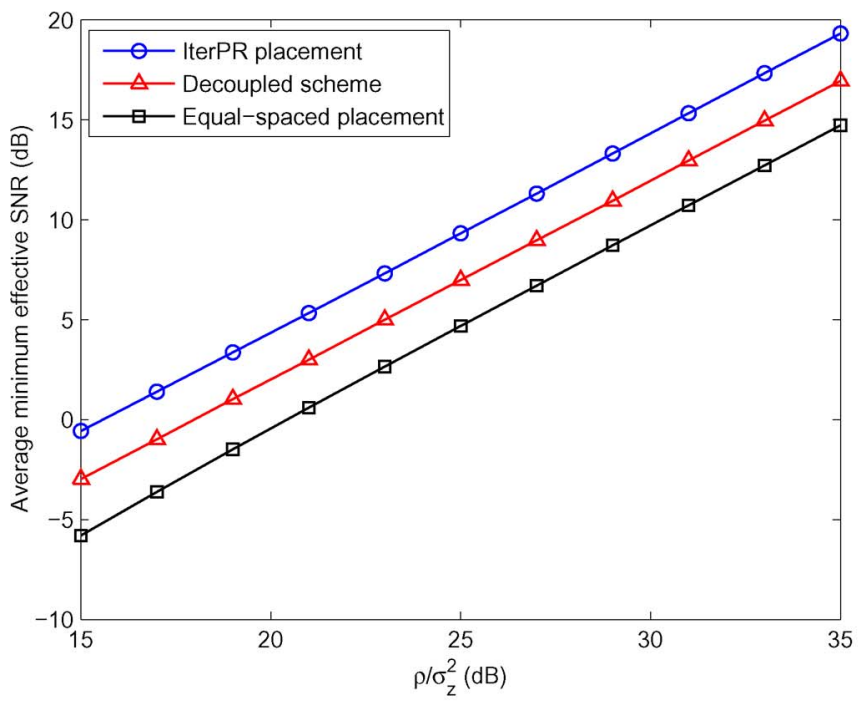

(a)

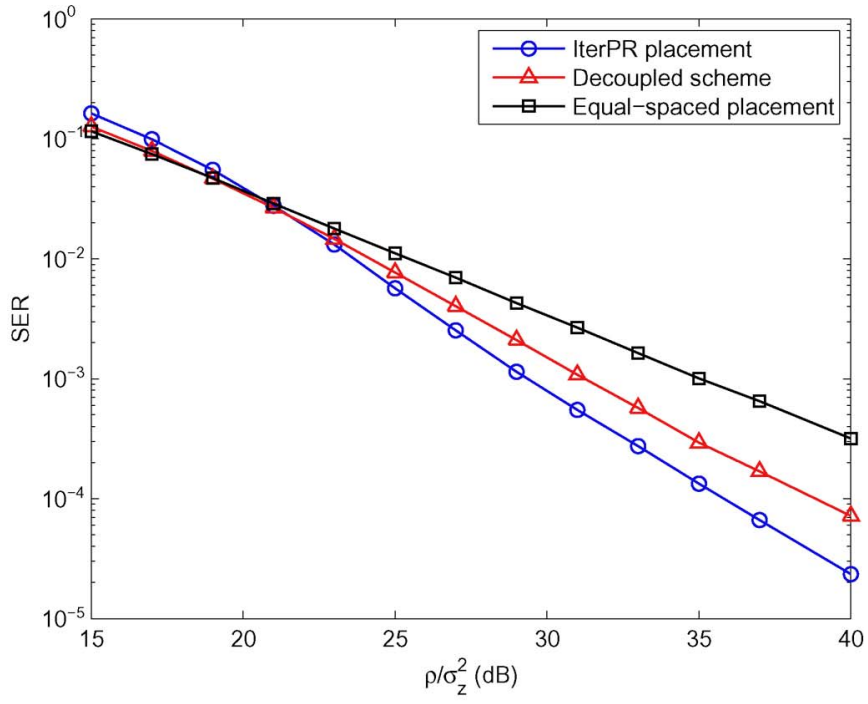

(b)

Fig. 6. Performances of different pilot placement schemes with $N=64, N_{p}=8, L_{1}=6$, and $L_{2}=4$. (a) Minimum effective SNR. (b) SER.

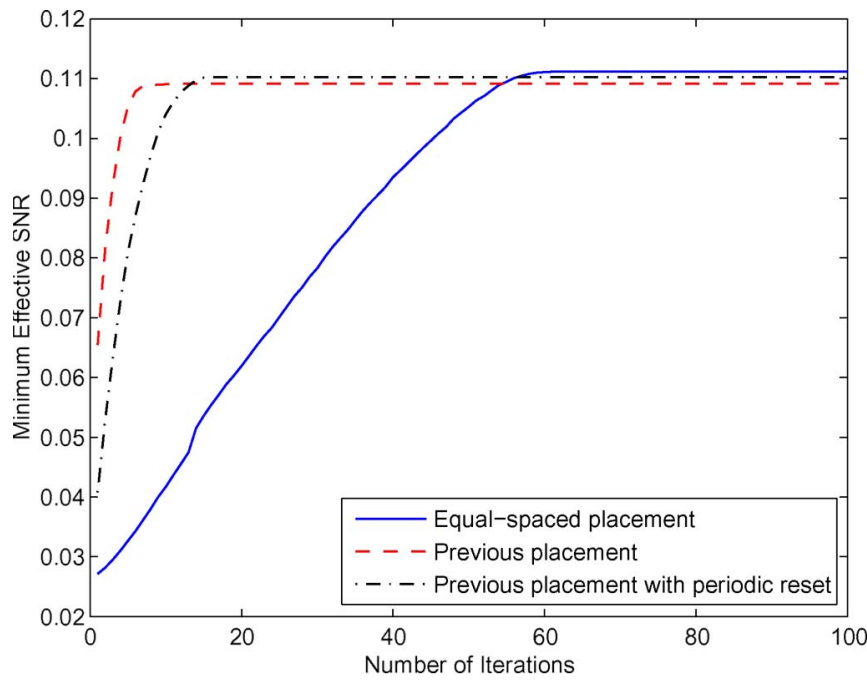

Fig. 7. Minimum effective SNR versus the number of iterations for different initial placements including the equal-spaced placement, the previous placement, and the previous one with reset to the equal-spaced one every five executions of the IterPR algorithm. Here, $N=1024$, and $N_{p}=64$.

method yields a slight loss in performance but can be overcome by resetting the initial pilot placement to the equal-spaced scheme every several number of updates, referred to as the "periodic reset" scheme in Fig. 7. The frequency of the periodic reset can be chosen to exploit the tradeoff between convergence speed and performance.

In Figs. 8 and 9, we further consider relay systems that employ SP. For all schemes, the SP function is chosen to be the inverse mapping between the SR and the RD subcarriers according to their respective effective SNRs. Notice that, when $\mathrm{SP}$ is employed, the pilot subcarriers on the SR and RD links can be chosen separately. Therefore, in the decoupled scheme, the pilot subcarriers will also be chosen separately on the SR and RD links according to their respective effective SNRs, i.e., $\left|\hat{H}_{\mathrm{SR}, k}\right|^{2} /\left(1+\gamma_{d} \epsilon_{\mathrm{SR}, k}\right)$ and $\left|\hat{H}_{\mathrm{RD}, k}\right|^{2} /\left(1+\gamma_{d} \epsilon_{\mathrm{RD}, k}\right)$. Specifically, in Fig. 8, where $N=16$ and $N_{p}=4$, we can see that the IterPR-SP scheme also performs close to the optimal scheme,

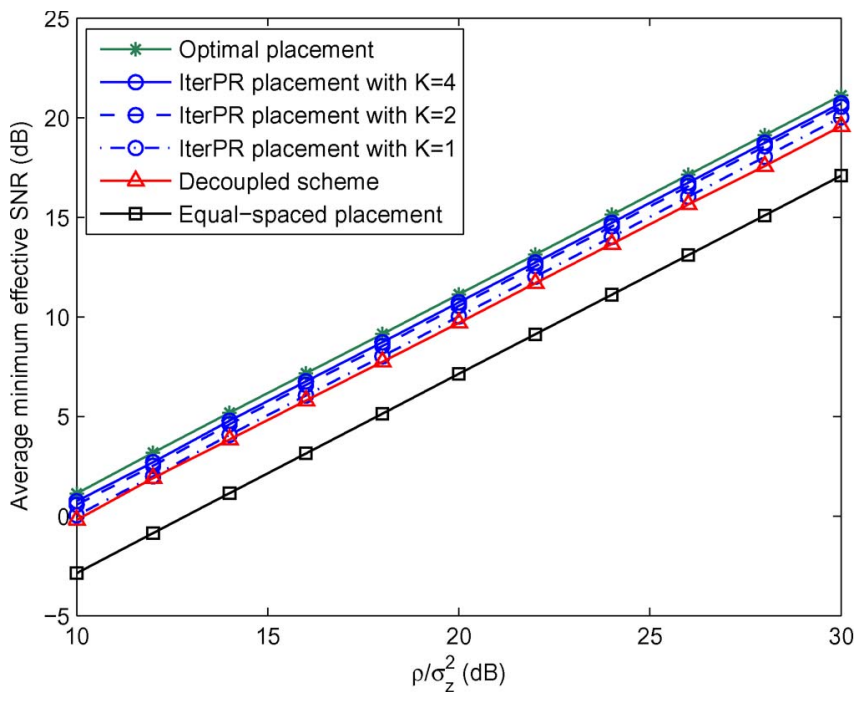

Fig. 8. Average minimum effective SNR of different pilot placement schemes for systems with SP and with $N=16, N_{p}=4, L_{1}=3$, and $L_{2}=2$.

similar to the case without SP. For the IterPR-SP scheme, the improvement in average minimum effective SNR also falls below $0.1 \%$ after only $K=4$ iterations. In Fig. 9, we further consider a large-scale system with $N=64$ and $N_{p}=8$. We can see that the IterPR-SP scheme (with $K=8$ ) significantly outperforms the other schemes in terms of the average minimum effective SNR and SER. By comparing with Fig. 6, we can see that the advantages of the feedback-aided pilot placement policies are even more pronounced in systems with SP.

In Fig. 10, the feedback gain obtained with the proposed IterPR scheme is shown with respect to the number of pilot subcarriers, i.e., $N_{p}$. We can see that the feedback gain increases with the number of pilot subcarriers for systems both with and without SP. In fact, the increase is larger for systems with SP. This is because frequency diversity is more evident in the case with SP, and thus, whether or not the channel is accurately estimated becomes important. In Fig. 11, the feedback gain of the proposed IterPR scheme is shown for channels with 


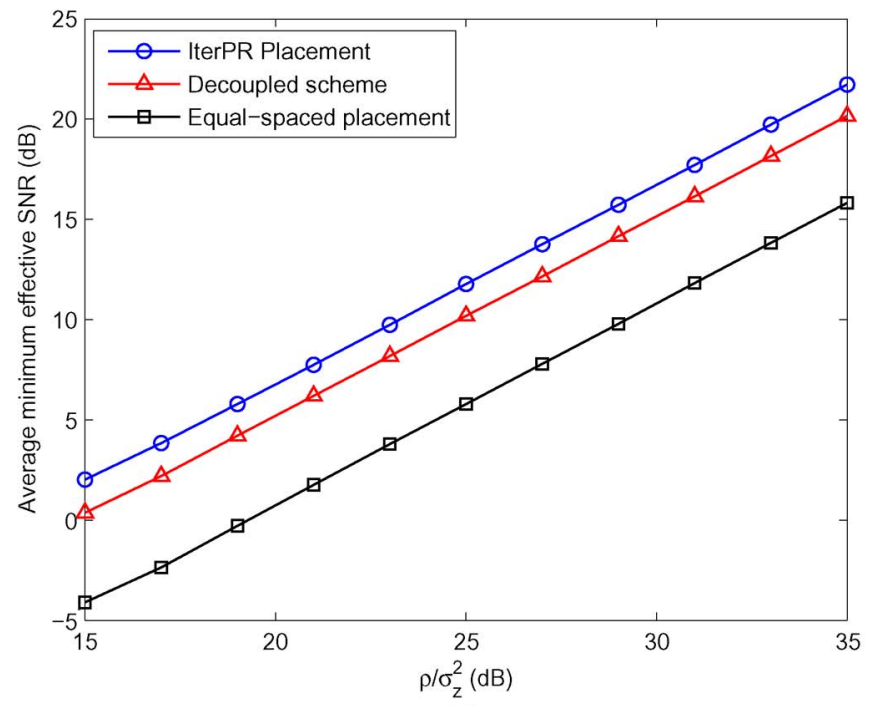

(a)

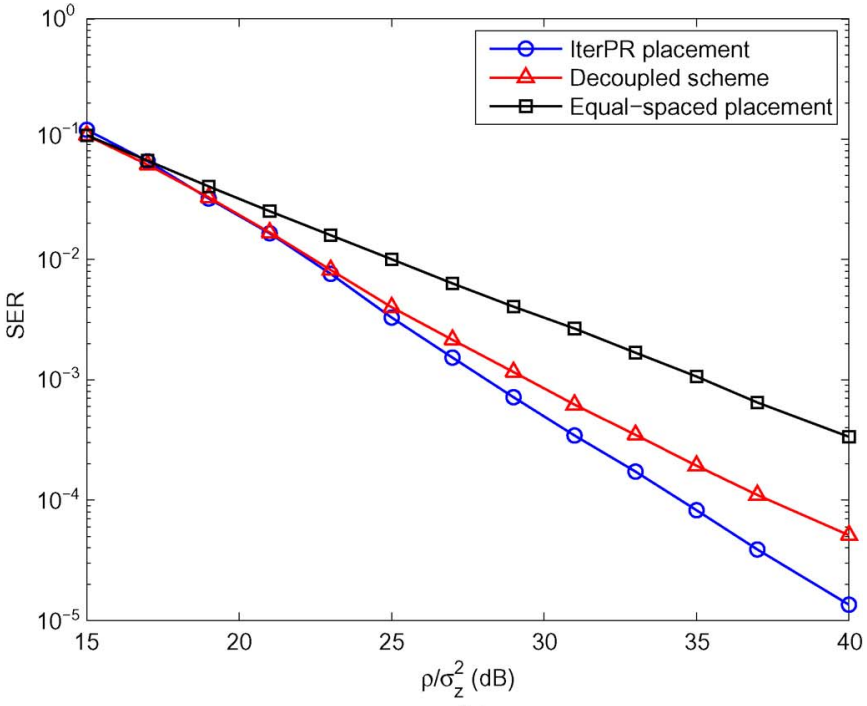

(b)

Fig. 9. Comparison of pilot placement schemes for systems with SP and $N=64, N_{p}=8, L_{1}=6$, and $L_{2}=4$. (a) Minimum effective SNR. (b) SER.

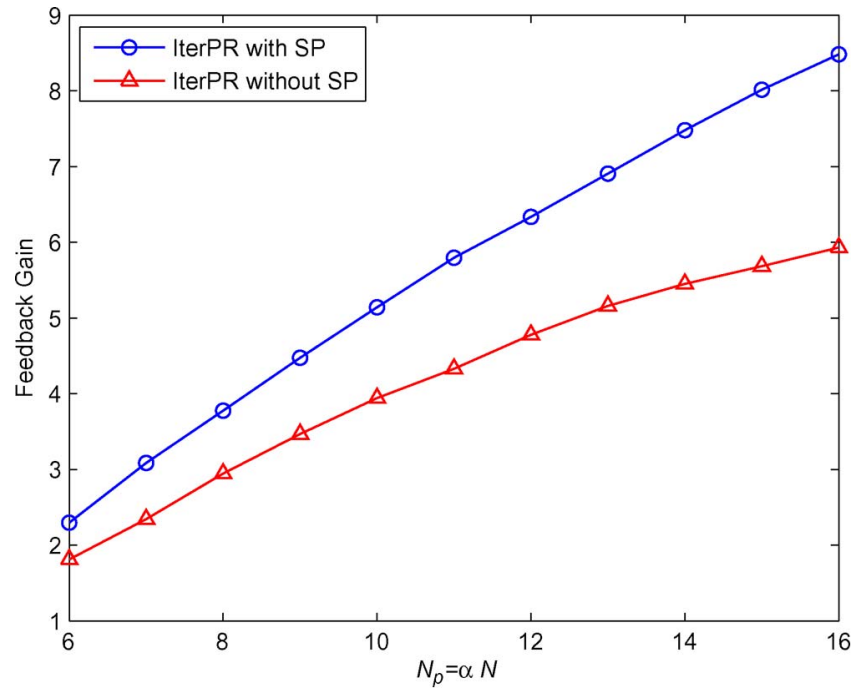

Fig. 10. Feedback gain of the proposed IterPR scheme for a different number of pilot subcarriers. Note that $N=64, L_{1}=6, L_{2}=4$, and $\rho / \sigma_{z}^{2}=20 \mathrm{~dB}$, and the number of iterations for the IterPR scheme $K=8$.

different channel lengths, i.e., $L_{1}$ and $L_{2}$. Note that larger channel lengths lead to higher frequency selectivity. We can observe that the feedback gain is larger for cases with higher frequency selectivity, and the improvement is more evident for systems with SP.

\section{CONCLUSION}

Feedback-aided pilot placement schemes for OFDM AF relay channels have been examined in this paper. The effective SNRs at the receivers and the analytic LBs of the achievable feedback gains were derived. The feedback gain with respect to the equal-spaced pilot placement scheme was shown to increase with the number of pilot symbols. Due to the complexity of the optimal policy, a suboptimal IterPR scheme that utilizes iterative exchanges between each pilot subcarrier and the data subcarrier with the minimum effective SNR was proposed. The studies were conducted for systems without and with SP.

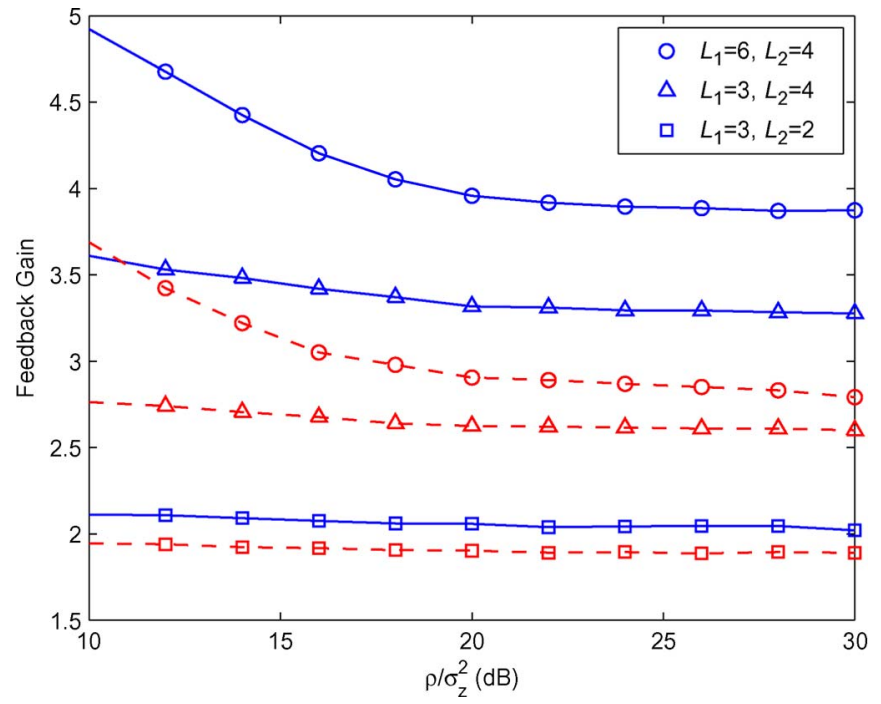

Fig. 11. Feedback gain of the proposed IterPR scheme for channels with different values of $L_{1}$ and $L_{2}$. The solid lines are for systems with SP, and the dashed lines are for those without. Here, we set $N=64, N_{p}=8$, and $K=8$.

In particular, for systems with SP, the optimal SP scheme was derived and shown to be an inverse mapping between the SR and the RD effective channel gains. The effectiveness of the proposed IterPR algorithms was demonstrated through numerical simulations.

\section{APPENDIX A}

\section{DERIVATION OF (8)}

In this paper, the channel estimates, i.e., $\hat{\mathbf{H}}_{\mathrm{SR}}$ and $\hat{\mathbf{H}}_{\mathrm{RD}}$, are used for channel equalization at the destination. The received signal after channel equalization for the data transmitted on subcarrier $k \in \mathcal{D}_{S}$ can thus be written as

$$
\begin{aligned}
\bar{Y}_{k} & =\hat{H}_{\mathrm{SR}, k}^{*} \hat{H}_{\mathrm{RD}, \varphi(k)}^{*} Y_{\mathrm{D}, \varphi(k)} \\
& =\Lambda_{\varphi(k)} s_{k}\left|\hat{H}_{\mathrm{SR}, k}\right|^{2}\left|\hat{H}_{\mathrm{RD}, \varphi(k)}\right|^{2}+V_{1, k}+V_{2, k}
\end{aligned}
$$


where

$$
\begin{aligned}
& V_{1, k}=\Lambda_{\varphi(k)} s_{k}\left(\hat{H}_{\mathrm{SR}, k}^{*} \hat{H}_{\mathrm{RD}, \varphi(k)}^{*} \tilde{H}_{\mathrm{SR}, k} \tilde{H}_{\mathrm{RD}, \varphi(k)}\right. \\
& \left.\quad+\left|\hat{H}_{\mathrm{SR}, k}\right|^{2} \hat{H}_{\mathrm{RD}, \varphi(k)}^{*} \tilde{H}_{\mathrm{RD}, \varphi(k)}+\hat{H}_{\mathrm{SR}, k}^{*}\left|\hat{H}_{\mathrm{RD}, \varphi(k)}\right|^{2} \tilde{H}_{\mathrm{SR}, k}\right) \\
& V_{2, k}=\Lambda_{\varphi(k)} \hat{H}_{\mathrm{SR}, k}^{*} \hat{H}_{\mathrm{RD}, \varphi(k)}^{*} \tilde{H}_{\mathrm{RD}, \varphi(k)} Z_{\mathrm{R}, k} \\
& \quad+\Lambda_{\varphi(k)} \hat{H}_{\mathrm{SR}, k}^{*}\left|\hat{H}_{\mathrm{RD}, \varphi(k)}\right|^{2} Z_{\mathrm{R}, k}+\hat{H}_{\mathrm{SR}, k}^{*} \hat{H}_{\mathrm{RD}, \varphi(k)}^{*} Z_{\mathrm{D}, \varphi(k)}
\end{aligned}
$$

The signal part of $\bar{Y}_{k}$ has average power given by $P_{d}=$ $\Lambda_{\varphi(k)}^{2} \rho_{d}\left|\hat{H}_{\mathrm{SR}, k}\right|^{4}\left|\hat{H}_{\mathrm{RD}, \varphi(k)}\right|^{4}$, and the noise part has average power given by

$$
\begin{aligned}
P_{v}= & \mathrm{E}\left\{\left(V_{1, k}+V_{2, k}\right)\left(V_{1, k}^{*}+V_{2, k}^{*}\right)\right\} \\
= & \Lambda_{\varphi(k)}^{2} \rho_{d}\left|\hat{H}_{\mathrm{SR}, k}\right|^{4}\left|\hat{H}_{\mathrm{RD}, \varphi(k)}\right|^{2} \epsilon_{\mathrm{RD}, \varphi(k)} \\
& +\Lambda_{\varphi(k)}^{2} \rho_{d}\left|\hat{H}_{\mathrm{SR}, k}\right|^{2}\left|\hat{H}_{\mathrm{RD}, \varphi(k)}\right|^{4} \epsilon_{\mathrm{SR}, k} \\
& +\Lambda_{\varphi(k)}^{2} \rho_{d}\left|\hat{H}_{\mathrm{SR}, k}\right|^{2}\left|\hat{H}_{\mathrm{RD}, \varphi(k)}\right|^{2} \epsilon_{\mathrm{SR}, k} \epsilon_{\mathrm{RD}, \varphi(k)} \\
& +\Lambda_{\varphi(k)}^{2} \sigma_{z}^{2}\left|\hat{H}_{\mathrm{SR}, k}\right|^{2}\left|\hat{H}_{\mathrm{RD}, \varphi(k)}\right|^{4} \\
& +\Lambda_{\varphi(k)}^{2} \sigma_{z}^{2}\left|\hat{H}_{\mathrm{SR}, k}\right|^{2}\left|\hat{H}_{\mathrm{RD}, \varphi(k)}\right|^{2} \epsilon_{\mathrm{RD}, \varphi(k)} \\
& +\sigma_{z}^{2}\left|\hat{H}_{\mathrm{SR}, k}\right|^{2}\left|\hat{H}_{\mathrm{RD}, \varphi(k)}\right|^{2} .
\end{aligned}
$$

Thus, the effective SNR, defined by $\xi_{k} \triangleq P_{d} / P_{v}$, can be computed as in (36), shown at the bottom of the next page, where $1 / \Lambda_{\varphi(k)}^{2}=\left|\hat{H}_{\mathrm{SR}, k}\right|^{2}+\epsilon_{\mathrm{SR}, k}+\left(1 / \gamma_{d}\right)$, and $\zeta_{k}$ is given in (9).

\section{APPENDIX B}

\section{DERIVATION OF (23)}

Given the pdf of $G(\beta)_{k}$ in (22), the cumulative density function (cdf) can be computed as

$$
\begin{aligned}
F_{G(\beta)}(g) \simeq & \int_{0}^{g} \frac{1}{0.625} \cdot\left(\varepsilon_{1}(\beta)+\varepsilon_{2}(\beta)\right) \\
& \cdot e^{-\frac{1}{0.625} \cdot x \cdot\left(\varepsilon_{1}(\beta)+\varepsilon_{2}(\beta)\right)} d x \\
= & 1-e^{-\frac{1}{0.625} \cdot g \cdot\left(\varepsilon_{1}(\beta)+\varepsilon_{2}(\beta)\right)} u(g)
\end{aligned}
$$

for all $k$. Note that (22) and (37) are also pdfs and cdfs of $\tilde{G}(\beta)_{k}$ for all $k$. Thus, the order statistics of $\tilde{G}(\beta)_{k}$ can then be computed as [42]

$$
\begin{aligned}
f_{\tilde{G}(\beta)_{r: n}}(g)= & \frac{n !}{(r-1) !(n-r) !} \cdot\left(F_{G(\beta)}(g)\right)^{r-1} \\
& \times\left(1-F_{G(\beta)}(g)\right)^{n-r} \cdot f_{G(\beta)}(g) \\
\simeq & \frac{\left(\varepsilon_{1}(\beta)+\varepsilon_{2}(\beta)\right) \cdot n !}{0.625 \cdot(r-1) !(n-r) !} \\
& \cdot \sum_{i=0}^{r-1} \frac{(r-1) !}{i !(r-1-i) !}(-1)^{i} \\
& \times e^{-\frac{1}{0.625} \cdot g \cdot(i+n-r+1)\left(\varepsilon_{1}(\beta)+\varepsilon_{2}(\beta)\right)} u(g) .
\end{aligned}
$$

The expectation of the $r$ th-order statistic $\tilde{G}(\beta)_{r: n}$ is then given by

$$
\begin{aligned}
\mathrm{E}\left[\tilde{G}(\beta)_{r: n}\right]= & \int_{0}^{\infty} g f_{\tilde{G}(\beta)_{r: n}}(g) d g \\
\simeq & \frac{\left(\varepsilon_{1}(\beta)+\varepsilon_{2}(\beta)\right)}{0.625} \cdot \frac{n !}{(r-1) !(n-r) !} \\
& \cdot \sum_{i=0}^{r-1} \frac{(r-1) !}{i !(r-1-i) !}(-1)^{i} \\
& \times \int_{0}^{\infty} g e^{-\frac{1}{0.625} \cdot g \cdot(i+n-r+1)\left(\varepsilon_{1}(\beta)+\varepsilon_{2}(\beta)\right)} d g \\
= & \frac{0.625}{\left(\varepsilon_{1}(\beta)+\varepsilon_{2}(\beta)\right)} \cdot \frac{n !}{(r-1) !(n-r) !} \\
& \times \sum_{i=0}^{r-1} \frac{(r-1) !}{i !(r-1-i) !} \frac{(-1)^{i}}{(i+n-r+1)^{2}} .
\end{aligned}
$$

\section{APPENDIX C}

\section{DERIVATION OF (25)}

To obtain (25), let us first prove the following lemma.

Lemma 1: For $p=2, \ldots, r$, we have

$$
\sum_{i=0}^{r}\left(\begin{array}{l}
r \\
i
\end{array}\right) i^{p} y^{i}=p !\left(\begin{array}{l}
r \\
p
\end{array}\right) y^{p}(1+y)^{r-p}+(1+y)^{r-p+1} g(y)
$$

where $g(y)$ is a polynomial of degree $(p-1)$.

Proof: Recall that, by taking the derivative of $\sum_{i=0}^{r}\left(\begin{array}{c}r \\ i\end{array}\right) y^{i}=$ $(1+y)^{r}$ and by multiplying both sides by $y$, we can show that $\sum_{i=0}^{r}\left(\begin{array}{c}r \\ i\end{array}\right) i y^{i}=r y(1+y)^{r-1}$. Using this fact, the lemma can be proven by induction as follows.

1) To show for $p=2$, let us again take the derivative of the foregoing equation and multiply both sides

$$
\begin{aligned}
\xi_{k} & =\frac{\gamma_{d} \cdot\left|\hat{H}_{\mathrm{SR}, k}\right|^{2}\left|\hat{H}_{\mathrm{RD}, \varphi(k)}\right|^{2}}{\gamma_{d}\left(\left|\hat{H}_{\mathrm{SR}, k}\right|^{2} \epsilon_{\mathrm{RD}, \varphi(k)}+\left|\hat{H}_{\mathrm{RD}, \varphi(k)}\right|^{2} \epsilon_{\mathrm{SR}, k}+\epsilon_{\mathrm{SR}, k} \epsilon_{\mathrm{RD}, \varphi(k)}\right)+\left|\hat{H}_{\mathrm{RD}, \varphi(k)}\right|^{2}+\epsilon_{\mathrm{RD}, \varphi(k)}+1 / \Lambda_{\varphi(k)}^{2}} \\
& =\frac{\gamma_{d} \cdot\left|\hat{H}_{\mathrm{SR}, k}\right|^{2}\left|\hat{H}_{\mathrm{RD}, \varphi(k)}\right|^{2}}{\zeta_{k}+\left|\hat{H}_{\mathrm{RD}, k}\right|^{2}+\left|\hat{H}_{\mathrm{RD}, k}\right|^{2}+\frac{1}{\gamma_{d}}}
\end{aligned}
$$


by $y$ to obtain $\sum_{i=0}^{r}\left(\begin{array}{c}r \\ i\end{array}\right) i^{2} y^{i}=r(r-1) y^{2}(1+y)^{r-2}+$ $r y(1+y)^{r-1}=2 !\left(\begin{array}{c}r \\ 2\end{array}\right) y^{2}(1+y)^{r-2}+(1+y)^{r-1} g(y)$, where $g(y)=r y$ is a polynomial of degree 1 .

2) Suppose that the statement holds for $p=k \geq 2$. That is

$$
\sum_{i=0}^{r}\left(\begin{array}{l}
r \\
i
\end{array}\right) i^{k} y^{i}=k !\left(\begin{array}{l}
r \\
k
\end{array}\right) y^{k}(1+y)^{r-k}+(1+y)^{r-k+1} g(y)
$$

where $g(y)$ is a polynomial of degree $k-1$. Then, by taking the derivative of the foregoing equation and multiplying both sides by $y$, we get

$$
\begin{aligned}
y & \left(\frac{d}{d y} \sum_{i=0}^{r}\left(\begin{array}{l}
r \\
i
\end{array}\right) i^{k} y^{i}\right) \\
= & \sum_{i=0}^{r}\left(\begin{array}{l}
r \\
i
\end{array}\right) i^{k+1} y^{i} \\
= & (r-k) k !\left(\begin{array}{l}
r \\
k
\end{array}\right) y^{k+1}(1+y)^{r-k-1}+k \cdot k !\left(\begin{array}{l}
r \\
k
\end{array}\right) y^{k}(1+y)^{r-k} \\
& +(r-k+1) \cdot(1+y)^{r-k} \cdot y \cdot g(y)+(1+y)^{r-k+1} \cdot y \cdot g^{\prime}(y) \\
= & (k+1) !\left(\begin{array}{c}
r \\
k+1
\end{array}\right) y^{k+1}(1+y)^{r-k-1}+(1+y)^{r-k} \cdot \tilde{g}(y)
\end{aligned}
$$

where $\tilde{g}(y)=k \cdot k !\left(\begin{array}{l}r \\ k\end{array}\right) y^{k}+(r-k+1) \cdot y \cdot g(y)+(1+y)$. $y \cdot g^{\prime}(y)$ is a polynomial of degree $k$. This shows that the statement also holds for $p=k+1$, and thus, the lemma is proved.

Using Lemma 1, we can then show that

$$
\left(\begin{array}{l}
n \\
r
\end{array}\right) \sum_{i=0}^{r}\left(\begin{array}{l}
r \\
i
\end{array}\right) \frac{(-1)^{i}}{\left(1+\frac{i}{n-r}\right)^{2}} \approx(r+1)\left(1+\frac{1}{n-r}\right)^{r}
$$

for $n \gg r$. To show this, let us first note that the term $1 /(1+i /(n-r))^{2}$ can be expanded as $1 /(1+i /(n-r))^{2}=$ $\sum_{k=0}^{\infty}(-1)^{k}(k+1)(i /(n-r))^{k}$. In this case, it follows that

$$
\begin{aligned}
& \left(\begin{array}{l}
n \\
r
\end{array}\right) \sum_{i=0}^{r}\left(\begin{array}{l}
r \\
i
\end{array}\right) \frac{(-1)^{i}}{\left(1+\frac{i}{n-r}\right)^{2}} \\
& =\left(\begin{array}{l}
n \\
r
\end{array}\right) \sum_{k=0}^{\infty} \frac{(-1)^{k}(k+1)}{(n-r)^{k}} \sum_{i=0}^{r}\left(\begin{array}{l}
r \\
i
\end{array}\right) i^{k}(-1)^{i} \\
& \stackrel{(a)}{=}\left(\begin{array}{l}
n \\
r
\end{array}\right) \sum_{k=r}^{\infty} \frac{(-1)^{k}(k+1)}{(n-r)^{k}} \sum_{i=0}^{r}\left(\begin{array}{l}
r \\
i
\end{array}\right) i^{k}(-1)^{i}
\end{aligned}
$$

where $(a)$ follows from Lemma 1 . Then, for $n \gg r$, we can approximate (38) by

$$
\begin{aligned}
\left(\begin{array}{l}
n \\
r
\end{array}\right) & \frac{(-1)^{r}(r+1)}{(n-r)^{r}} \sum_{i=0}^{r}\left(\begin{array}{l}
r \\
i
\end{array}\right) i^{r}(-1)^{i} \\
& \stackrel{(b)}{=} r !\left(\begin{array}{l}
n \\
r
\end{array}\right) \frac{(r+1)}{(n-r)^{r}} \geq(r+1)\left(1+\frac{1}{n-r}\right)^{r}
\end{aligned}
$$

where (b) also follows from Lemma 1. By applying (39) to (24) with $n=\tilde{N}$ and $r=\tilde{N}_{p}$, we obtain the approximate LB in (25).

\section{APPENDIX D}

\section{ProOF OF PROPOSITION 1}

Let $\mathcal{A}_{n}=\left\{a_{0}, \ldots, a_{n-1}\right\}$ and $\mathcal{B}_{n}=\left\{b_{0}, \ldots, b_{n-1}\right\}$ be sets consisting of $n$ positive real numbers, where $a_{0} \leq a_{1} \leq \cdots \leq$ $a_{n-1}$ and $b_{0} \leq b_{1} \leq \cdots \leq b_{n-1}$, and let $\varphi_{n}^{*}: \mathcal{A}_{n} \rightarrow \mathcal{B}_{n}$ be the inverse mapping between the two sets defined by $\varphi_{n}^{*}\left(a_{i}\right)=$ $b_{n-1-i}$ for $i=0, \ldots, n-1$. Proposition 1 can be proven by induction as follows.

1) For $n=2$, only two possible mappings can be found: 1) the inverse mapping $\varphi_{2}^{*}$ and 2) the mapping $\varphi_{2}^{\prime}$ : $\mathcal{A}_{2} \rightarrow \mathcal{B}_{2}$ defined by $\varphi_{2}^{\prime}\left(a_{0}\right)=b_{0}$ and $\varphi_{2}^{\prime}\left(a_{1}\right)=b_{1}$. Since $f\left(a_{0}, b_{0}\right) \leq f\left(a_{0}, b_{1}\right)$ and $f\left(a_{0}, b_{0}\right) \leq f\left(a_{1}, b_{0}\right)$, it follows that $\min \left\{f\left(a_{0}, \varphi_{2}^{\prime}\left(a_{0}\right)\right), f\left(a_{1}, \varphi_{2}^{\prime}\left(a_{1}\right)\right)\right\}=$ $f\left(a_{0}, b_{0}\right) \leq \min \left\{f\left(a_{0}, \varphi_{2}^{*}\left(a_{0}\right)\right), f\left(a_{1}, \varphi_{2}^{*}\left(a_{1}\right)\right)\right\}$. Hence, the inverse mapping $\varphi_{2}^{*}$ is optimal for $n=2$.

2) Suppose that the inverse mapping $\varphi_{k}^{*}: \mathcal{A}_{k} \rightarrow \mathcal{B}_{k}$ is optimal for some $k \geq 2$. We need to show that the inverse mapping $\varphi_{n}^{*}: \mathcal{A}_{n} \rightarrow \mathcal{B}_{n}$ is optimal for $n=k+1$.

Given the mapping for $a_{0}$ in the case of $n=k+1$, the best mapping (i.e., the mapping that yields the max-min value of $f$ ) for the remaining $k$ elements in each set is given by the inverse mapping. In this case, we define $\varphi_{k+1}^{(j)}: \mathcal{A}_{k+1} \rightarrow \mathcal{B}_{k+1}$ as the mapping, where $a_{0}$ is assigned to $b_{k-j}$, i.e., $\varphi_{k+1}^{(j)}\left(a_{0}\right)=$ $b_{k-j}$, whereas the remaining elements are given by the inverse mapping, i.e.,

$$
\varphi_{k+1}^{(j)}\left(a_{i}\right)= \begin{cases}b_{k+1-i}, & \text { for } 0<i \leq j \\ b_{k-i}, & \text { for } i>j .\end{cases}
$$

Notice that $\varphi_{k+1}^{(j)}$ is the best mapping among all those that assign $a_{0}$ to $b_{k-j}$ and that $\varphi_{k+1}^{(0)} \equiv \varphi_{k+1}^{*}$. We need to show that $\min _{i=0, \ldots, k} f\left(a_{i}, \varphi_{k+1}^{(0)}\left(a_{i}\right)\right) \geq \min _{i=0, \ldots, k} f\left(a_{i}, \varphi_{k+1}^{(j)}\left(a_{i}\right)\right)$ for any $j$.

Let $i_{0}=\arg \min _{i=0, \ldots, k} f\left(a_{i}, \varphi_{k+1}^{(0)}\left(a_{i}\right)\right)=\arg \min _{i=0, \ldots, k}$ $f\left(a_{i}, b_{k-i}\right)$ be the index of the pair that yields the minimum value of $f$ under inverse mapping. For any $j>$ 0 , it is easy to show that $f\left(a_{0}, \varphi_{k+1}^{(j)}\left(a_{0}\right)\right)=f\left(a_{0}, b_{k-j}\right) \leq$ $f\left(a_{0}, b_{k}\right)=f\left(a_{0}, \varphi_{k+1}^{(0)}\left(a_{0}\right)\right), \quad f\left(a_{i}, \varphi_{k+1}^{(j)}\left(a_{i}\right)\right)=f\left(a_{i}, b_{k+1-i}\right) \geq$ $f\left(a_{i}, b_{k-i}\right)=f\left(a_{i}, \varphi_{k+1}^{(0)}\left(a_{i}\right)\right)$ for $i=1, \ldots, j$ and $f\left(a_{i}\right.$, $\left.\varphi_{k+1}^{(j)}\left(a_{i}\right)\right)=f\left(a_{i}, \varphi_{k+1}^{(0)}\left(a_{i}\right)\right)$ for $i=j+1, \ldots, k$. It follows that if $j<i_{0}$, then

$$
\begin{array}{r}
\min _{i=0, \ldots, k} f\left(a_{i}, \varphi_{k+1}^{(j)}\left(a_{i}\right)\right)=\min \left\{f\left(a_{0}, b_{k-j}\right), f\left(a_{i_{0}}, b_{k-i_{0}}\right)\right\} \\
\leq f\left(a_{i_{0}}, b_{k-i_{0}}\right)=\min _{i=0, \ldots, k} f\left(a_{i}, \varphi_{k+1}^{(0)}\left(a_{i}\right)\right) .
\end{array}
$$

If $j \geq i_{0}$, then

$$
\begin{aligned}
& \min _{i=0, \ldots, k} f\left(a_{i}, \varphi_{k+1}^{(j)}\left(a_{i}\right)\right)=f\left(a_{0}, b_{k-j}\right) \\
& \quad \leq f\left(a_{i_{0}}, b_{k-i_{0}}\right)=\min _{i=0, \ldots, k} f\left(a_{i}, \varphi_{k+1}^{(0)}\left(a_{i}\right)\right) .
\end{aligned}
$$

Hence, the inverse mapping $\varphi_{k+1}^{(0)}$ is optimal for $n=k+1$. 


\section{APPENDIX E}

\section{DERIVATION OF (31)}

To obtain the result in (31), we first show the following two lemmas.

Lemma 2: With $f_{1}(i) \triangleq-a \cdot \ln \left(1-(2 i-1) /\left(2 \tilde{N}_{d}\right)\right)-$ $b \cdot \ln \left((2 i-1) /\left(2 \tilde{N}_{d}\right)\right)$, where $\tilde{N}_{d} \geq 1$, we have

$$
\max _{i \in\left\{1, \ldots, \tilde{N}_{d}\right\}} f_{1}(i)= \begin{cases}f_{1}(1), & \text { for } a \leq b \\ f_{1}\left(\tilde{N}_{d}\right), & \text { for } a>b\end{cases}
$$

Proof: Let $g(x)=-a \cdot \ln (1-x)-b \cdot \ln (x)$ for $0<x<$ 1 and take the derivative

$$
g^{\prime}(x)=\frac{d}{d x} g(x)=\frac{a}{1-x}-\frac{b}{x}=\frac{(a+b) x-b}{x(1-x)}
$$

Thus, it follows that $g^{\prime}(x) \leq 0$ for $0<x \leq b /(a+b)$ and that $g^{\prime}(x)>0$ for $b /(a+b)<x<1$. That is, $g(x)$ is decreasing with $x$ when $0<x \leq b /(a+b)$ and is increasing with $x$ when $b /(a+b)<x<1$. Hence, the maximum value of $f_{1}(i)$ is achieved with either $i=1$ or $i=\tilde{N}_{d}$. For $\tilde{N}_{d} \geq 1$, it holds that $-\ln \left(1 /\left(2 \tilde{N}_{d}\right)\right) \geq-\ln \left(1-1 /\left(2 \tilde{N}_{d}\right)\right)>0$. Thus, for $a \leq b, f(1)=-a \cdot \ln \left(1-1 /\left(2 \tilde{N}_{d}\right)\right)-b \cdot \ln \left(1 /\left(2 \tilde{N}_{d}\right)\right) \geq$ $-a \cdot \ln \left(1 /\left(2 \tilde{N}_{d}\right)\right)-b \cdot \ln \left(1-1 /\left(2 \tilde{N}_{d}\right)\right)=f_{1}\left(\tilde{N}_{d}\right)$ and vice versa. The lemma then follows.

Lemma 3: With $f_{2}(i) \triangleq \ln \left(1-(2 i-1) /\left(2 \tilde{N}_{d}\right)\right) \ln ((2 i-1) /$ $\left.\left(2 \tilde{N}_{d}\right)\right)$, we have

$$
\min _{i \in\left\{1, \ldots, \tilde{N}_{d}\right\}} f_{2}(i)=f_{2}(1)=f_{2}\left(\tilde{N}_{d}\right) .
$$

Proof: Let $g(x)=\ln (1-x) \ln (x)$ for $0<x<1$. By taking the derivative of $g(x)$, we get

$g^{\prime}(x)=\frac{d}{d x} g(x)=\frac{1}{x} \ln (1-x)-\frac{1}{1-x} \ln (x)=\ln \left(\frac{(1-x)^{\frac{1}{x}}}{x^{\frac{1}{1-x}}}\right)$

Notice that, for $0<x \leq 1 / 2$, we have $1 / x \geq 2,1 / 2 \leq(1-$ $x)<1$, and $1<1 /(1-x) \leq 2$; thus, $0<x^{1 /(1-x)} \leq 1 / 4$, and $(1-x)^{1 / x} \geq 1 / 4$. By substituting these inequalities into the foregoing equation, it then follows that $g^{\prime}(x) \geq 0$ for $0<x \leq$ $1 / 2$ since $(1-x)^{1 / x} / x^{1 /(1-x)} \geq 1$. Similarly, we can show that $g^{\prime}(x)<0$ for $1 / 2<x<1$. That is, $g(x)$ increases with $x$ when $0<x \leq 1 / 2$ and decreases with $x$ when $1 / 2<x<1$. Since $0<(2 i-1) /\left(2 \tilde{N}_{d}\right)<1$ for $i=1, \ldots, \tilde{N}_{d}$, the minimum of $f_{2}(i)$ over the set $\left\{1, \ldots, \tilde{N}_{d}\right\}$ is achieved with either $i=1$ or $i=\tilde{N}_{d}$. The lemma then follows since $f(1)=f\left(\tilde{N}_{d}\right)=$ $\ln \left(1-1 /\left(2 \tilde{N}_{d}\right)\right) \ln \left(1 /\left(2 \tilde{N}_{d}\right)\right)$.

By Lemmas 2 and 3, it follows that

$$
\begin{aligned}
\min _{i \in\left\{1, \ldots, N_{d}\right\}} & \frac{\ln \left(1-\frac{2 i-1}{2 \tilde{N}_{d}}\right) \cdot \ln \left(\frac{2 i-1}{2 \tilde{N}_{d}}\right)}{-\sigma_{\mathrm{SR}}^{2} \cdot \ln \left(1-\frac{2 i-1}{2 \tilde{N}_{d}}\right)-\sigma_{\mathrm{RD}}^{2} \cdot \ln \left(\frac{2 i-1}{2 \tilde{N}_{d}}\right)} \\
= & \begin{cases}\frac{\ln \left(1-\frac{1}{2 \tilde{N}_{d}}\right) \cdot \ln \left(\frac{1}{2 \tilde{N}_{d}}\right)}{-\sigma_{\mathrm{SR}}^{2} \cdot \ln \left(1-\frac{1}{2 \tilde{N}_{d}}\right)-\sigma_{\mathrm{RD}}^{2} \cdot \ln \left(\frac{1}{2 \tilde{N}_{d}}\right),}, & \text { for } \sigma_{\mathrm{SR}}^{2} \leq \sigma_{\mathrm{RD}}^{2} \\
\frac{\ln \left(1-\frac{1}{2 \tilde{N}_{d}}\right) \cdot \ln \left(\frac{1}{2 \tilde{N}_{d}}\right)}{-\sigma_{\mathrm{SR}}^{2} \cdot \ln \left(\frac{1}{2 \tilde{N}_{d}}\right)-\sigma_{\mathrm{RD}}^{2} \cdot \ln \left(1-\frac{1}{2 \tilde{N}_{d}}\right)}, & \text { for } \sigma_{\mathrm{SR}}^{2}>\sigma_{\mathrm{RD}}^{2} .\end{cases}
\end{aligned}
$$

\section{REFERENCES}

[1] A. Sendonaris, E. Erkip, and B. Aazhang, "User cooperation diversityPart I: System description," IEEE Trans. Commun., vol. 51, no. 11, pp. 1927-1938, Nov. 2003

[2] J. N. Laneman, D. N. C. Tse, and G. W. Wornell, "Cooperative diversity in wireless networks: Efficient protocols and outage behavior," IEEE Trans. Inform. Theory, vol. 50, no. 12, pp. 3062-3080, Dec. 2004.

[3] Y.-W. P. Hong, W.-J. Huang, and C.-C. J. Kuo, Cooperative Communications and Networking: Technologies and System Design. Boston, MA: Springer-Verlag, 2010.

[4] T. E. Hunter and A. Nosratinia, "Diversity through coded cooperation," IEEE Trans. Wireless Commun., vol. 5, no. 2, pp. 283-289, Feb. 2006.

[5] G. Kramer, M. Gastpar, and P. Gupta, "Cooperative strategies and capacity theorems for relay networks," IEEE Trans. Inform. Theory, vol. 51, no. 9 , pp. 3037-3063, Sep. 2005

[6] Y. Yang, H. Hu, J. Xu, and G. Mao, "Relay technologies for WiMAX and LTE-advanced mobile systems," IEEE Commun. Mag., vol. 47, no. 10, pp. 100-105, Oct. 2009.

[7] T. Riihonen, R. Wichman, and S. Werner, "Evaluation of OFDM(A) relaying protocols: Capacity analysis in infrastructure framework," IEEE Trans. Veh. Technol., vol. 61, no. 1, pp. 360-374, Jan. 2012.

[8] I. Hammerstrom and A. Wittneben, "Power allocation schemes for amplify-and-forward MIMO-OFDM relay links," IEEE Trans. Wireless Commun., vol. 6, no. 8, pp. 2798-2802, Aug. 2007.

[9] A. Hottinen and T. Heikkinen, "Subchannel assignment in OFDM relay nodes," in Proc. 40th Annu. Conf. Inf. Sci. Syst., Princeton, NJ, Mar. 2006, pp. 1314-1317.

[10] A. Hottinen and T. Heikkinen, "Optimal subchannel assignment in a twohop OFDM relay," in Proc. IEEE 8th Workshop Signal Process. Adv. Wireless Commun., Helsinki, Finland, Jun. 2007, pp. 1-5.

[11] Y. Li, W. Wang, J. Kong, and M. Peng, "Subcarrier pairing for amplifyand-forward and decode-and-forward OFDM relay links," IEEE Commun. Lett., vol. 13, no. 4, pp. 209-211, Apr. 2009.

[12] T. Riihonen, R. Wichman, J. Hamalainen, and A. Hottinen, "Analysis of subcarrier pairing in a cellular OFDMA relay link," in Proc. Int. ITG Workshop Smart Antennas, Darmstadt, Germany, Feb. 2008, pp. 104-111.

[13] Z. Han, T. Himsoon, W. P. Siriwongpairat, and K. J. R. Liu, "Resource allocation for multiuser cooperative OFDM networks: Who helps whom and how to cooperate," IEEE Trans. Veh. Technol., vol. 58, no. 5, pp. 23762391, Jun. 2009.

[14] K.-Y. Sung, Y.-W. P. Hong, and C.-C. Chao, "Resource allocation and partner selection for cooperative multicarrier systems," IEEE Trans. Veh. Technol., vol. 60, no. 7, pp. 3228-3240, Sep. 2011.

[15] T. Wang and L. Vandendorpe, "Sum rate maximized resource allocation in multiple DF relays aided OFDM transmission," IEEE J. Sel. Areas Commun., vol. 29, no. 8, pp. 1559-1571, Sep. 2011.

[16] T. Wang and L. Vandendorpe, "WSR maximized resource allocation in multiple DF relays aided OFDMA downlink transmission," IEEE Trans. Signal Process., vol. 59, no. 8, pp. 3964-3976, Aug. 2011.

[17] T. Riihonen, R. Wichman, and S. Werner, "Capacity evaluation of DF protocols for OFDMA infrastructure relay links," in Proc. IEEE Global Commun. Conf., Honolulu, HI, Dec. 2009, pp. 1-6.

[18] T. Wang, "Weighted sum power minimisation for multichannel decodeand-forward relaying," IET Electron. Lett., vol. 48, no. 7, pp. 410-411, Mar. 2012.

[19] J. K. Cavers, "An analysis of pilot symbol assisted modulation for Rayleigh fading channels," IEEE Trans. Veh. Technol., vol. 40, no. 4, pp. 686-693, Nov. 1991.

[20] L. Tong, B. M. Sadler, and M. Dong, "Pilot-assisted wireless transmissions: General model, design criteria, and signal processing," IEEE Signal Process. Mag., vol. 21, no. 6, pp. 12-25, Nov. 2004.

[21] M. Dong and L. Tong, "Optimal design and placement of pilot symbols for channel estimation," IEEE Trans. Signal Process., vol. 50, no. 12, pp. 3055-3069, Dec. 2002.

[22] M. Dong, L. Tong, and B. M. Sadler, "Optimal insertion of pilot symbols for transmissions over time-varying flat fading channels," IEEE Trans. Signal Process., vol. 52, no. 5, pp. 1403-1418, May 2004.

[23] B. Hassibi and B. M. Hochwald, "How much training is needed in multiple-antenna wireless links?" IEEE Trans. Inform. Theory, vol. 49, no. 4, pp. 951-963, Apr. 2003.

[24] H. Vikalo, B. Hassibi, B. Hochwald, and T. Kailath, "On the capacity of frequency-selective channels in training-based transmission schemes," IEEE Trans. Signal Process., vol. 52, no. 9, pp. 2572-2583, Sep. 2004.

[25] S. Adireddy, L. Tong, and H. Viswanathan, "Optimal placement of training for frequency-selective block-fading channels," IEEE Trans. Inform. Theory, vol. 48, no. 8, pp. 2338-2353, Aug. 2002. 
[26] R. Negi and J. Cioffi, "Pilot tone selection for channel estimation in a mobile OFDM system," IEEE Trans. Consum. Electron., vol. 44, no. 3, pp. 1122-1128, Aug. 1998.

[27] S. Ohno and G. B. Giannakis, "Optimal training and redundant precoding for block transmissions with application to wireless OFDM," IEEE Trans. Commun., vol. 50, no. 12, pp. 2113-2123, Dec. 2002.

[28] X. Cai and G. B. Giannakis, "Error probability minimizing pilots for OFDM with M-PSK modulation over Rayleigh-fading channels," IEEE Trans. Veh. Technol., vol. 53, no. 1, pp. 146-155, Jan. 2004.

[29] A. Y. Panah, B. Nosrat-Makouei, and R. G. Vaughan, "Non-uniform pilot-symbol allocation for closed-loop OFDM," IEEE Trans. Wireless Commun., vol. 7, no. 7, pp. 2723-2731, Jul. 2008.

[30] A. Y. Panah, R. G. Vaughan, and R. W. Heath, Jr., "Optimizing pilot locations using feedback in OFDM systems," IEEE Trans. Veh. Technol., vol. 58, no. 6, pp. 2803-2814, Jul. 2009.

[31] F. Gao, T. Cui, and A. Nallanathan, "On channel estimation and optimal training design for amplify and forward relay networks," IEEE Trans. Wireless Commun., vol. 7, no. 5, pp. 1907-1916, May 2008.

[32] F. Gao, T. Cui, and A. Nallanathan, "Optimal training design for channel estimation in decode-and-forward relay networks with individual and total power constraints," IEEE Trans. Signal Process., vol. 56, no. 12, pp. 5937-5949, Dec. 2008.

[33] K.-Y. Sung, Y.-W. P. Hong, and C.-C. Chao, "Feedback-aided pilot placement for amplify-and-forward OFDM relay links," in Proc. IEEE 11th Int. Symp. Spread Spectr. Tech. Appl., Taichung, Taiwan, Oct. 2010, pp. 17-22.

[34] K.-Y. Sung, Y.-W. P. Hong, and C.-C. Chao, "Feedback-aided pilot placement for OFDM relay links with subcarrier pairing," in Proc. IEEE Int. Conf. Commun., Kyoto, Japan, Jun. 2011, pp. 1-5.

[35] M. Herdin, "A chunk base OFDM amplify-and-forward relaying scheme for 4G mobile radio systems," in Proc. IEEE Int. Conf. Commun., Istanbul, Turkey, Jun. 2006, pp. 4507-4512.

[36] C. R. N. Athaudage, M. Saito, and J. Evans, "Performance analysis of dual-hop OFDM relay systems with subcarrier pairing," in Proc. IEEE Int. Conf. Commun., Beijing, China, Jun. 2008, pp. 4419-4423.

[37] O. Amin, B. Gedik, and M. Uysal, "Channel estimation for amplifyand-forward relaying: Cascaded against disintegrated estimators," IET Commun., vol. 4, no. 10, pp. 1207-1216, Jul. 2010.

[38] S. M. Kay, Fundamentals of Statistical Signal Processing: Estimation Theory. Englewood Cliffs, NJ: Prentice-Hall, 1993.

[39] T. S. Rappaport, Wireless Communications: Principles and Practice. Upper Saddle River, NJ: Prentice-Hall, 2002.

[40] B. O'Hara and A. Petrick, The IEEE 802.11 Handbook: A Designer's Companion. New York: IEEE Press, 1999.

[41] W. Jakes, Mobile Microwave Communication. New York: Wiley, 1974.

[42] M. Ahsanullah and V. B. Nevzorov, Ordered Random Variables. Huntington, NY: Nova, 2001.

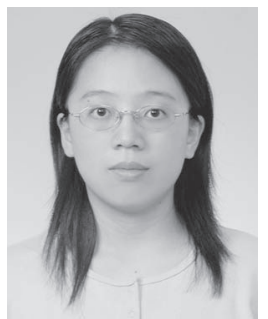

Kuang-Yu Sung (S'05) was born in Taipei, Taiwan, in 1980. She received the B.S. degree in electrical engineering in 2002 and the M.S. and Ph.D. degrees in communications engineering from the National Tsing Hua University, Hsinchu, Taiwan, in 2004 and 2011, respectively.

Since October 2011, she has been with the Institute of Communications Engineering, National Chiao Tung University, Hsinchu, where she is currently an Assistant Research Fellow. Her research interests include digital communications, signal processing, information theory, and cooperative communications.

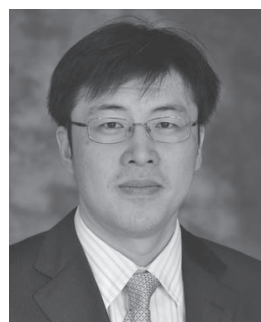

Y.-W. Peter Hong (S'01-M'05) received the B.S. degree in electrical engineering from the National Taiwan University, Taipei, Taiwan, in 1999 and the $\mathrm{Ph} . \mathrm{D}$. degree in electrical engineering from Cornell University, Ithaca, NY, in 2005.

$\mathrm{He}$ joined the Institute of Communications Engineering and the Department of Electrical Engineering, National Tsing Hua University, Hsinchu, Taiwan, in the Fall 2005, where he is currently an Associate Professor. He is a coauthor (with W.-J. Huang and C.-C. J. Kuo) of the book Cooperative Communications and Networking: Technologies and System Design (Springer, 2010). His research interests include cooperative communications, physical layer secrecy, distributed signal processing for sensor networks, and PHY-MAC cross-layer designs for wireless networks.

Prof. Hong received the Best Paper Award for Young Authors from the IEEE Information Theory/Communications Society Taipei/Tainan Chapter in 2005, the Best Paper Award among unclassified papers at the 2005 Military Communications Conference, the Junior Faculty Research Award from the College of Electrical Engineering and Computer Science (EECS) and from National Tsing Hua University in 2009 and 2010, respectively, and the Outstanding Teaching Award from the College of EECS in 2010. In 2010, he also received the IEEE Communications Society Asia-Pacific Outstanding Young Researcher Award. In 2011, he received the Y. Z. Hsu Scientific Paper Award and the Wu Ta-You Memorial Award. He is currently an Associate Editor for the IEEE TRANSACTIONS ON Signal PROCESSING and the IEEE TRANSACTIONS ON INFORMATION FORENSICS AND SECURITY.

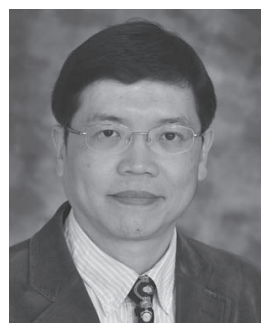

Chi-Chao Chao (S'87-M'89) was born in Taipei, Taiwan, in 1961. He received the B.S. degree in electrical engineering from the National Taiwan University, Taipei, in 1983 and the M.S. and Ph.D. degrees in electrical engineering from the California Institute of Technology (Caltech), Pasadena, in 1986 and 1989 , respectively.

He was an instructor with the Department of Weapon Command and Control Systems, Naval Weapon School, Kaohsiung, Taiwan, during his military service from 1983 to 1985 . Since September 1989, he has been with the National Tsing Hua University (NTHU), Hsinchu, Taiwan, where he is currently a Tsing Hua Distinguished Professor with the Department of Electrical Engineering and the Institute of Communications Engineering. He was the Chair of the Department of Electrical Engineering, NTHU, from February 2010 to July 2012, and the Director of the Institute of Communications Engineering, NTHU, from August 2005 to July 2008. He held visiting positions at Caltech from September 1995 to March 1996; at Bell Communications Research, Morristown, NJ, from March 1996 to August 1996; and at Osaka University, Japan, from April 2009 to September 2009. His current research interests include digital communications, error-correcting codes, information theory, and wireless networks.

Dr. Chao was the Secretary of the IEEE Taipei Section from 1997 to 1998, the Chair of the IEEE Information Theory Society Taipei Chapter from 1999 to 2001, and the Chair of the IEEE Communications Society Taipei Chapter from 2009 to 2010. He served as an Associate Editor for the IEEE Communications LetTers from 2002 to 2005. He was a General CoChair of the 2010 International Symposium on Information Theory and its Applications and the 2010 International Symposium on Spread Spectrum Techniques and Applications held in Taichung, Taiwan, October 2010. He received the Distinguished Teaching Award from NTHU in 1993, 2002, and 2008. 